\title{
ENERGY RELATED APPLICATIONS OF ELEMENTARY PARTICLE PHYSICS
}

Final Report

DOE/ER/13858--3

DE93 006983

\author{
Johann Rafelski \\ Department of Physics \\ University of Arizona. Tucson. AZ 85721
}

August 31, 1991

Prepared for

The US Department of Energy

AGREEMENT NO. DE-FG02-88ER13858

\begin{abstract}
NOTICE
This report was prepared as an account of work sponsored by the United States Government. Neither the United States nor the Department of Energy, nor any of their employees, nor any of their contractors, subcontractors, or their employees, makes any warranty, express or implied, or assumes any legal liability or responsibility for the eccuracy, completeness, or usefulness of any information, apparatus, product or process disclosed or represents that its use would not infringe privately-owned rights.
\end{abstract}

\section{DISCLAIMER}

This report was prepared as an account of work sponsored by an agency of the United States Government. Neither the United States Government nor any agency thereof, nor any of their employees, makes any warranty, express or implied, or assumes any legal liability or responsibility for the accuracy, completeness, or usefulness of any information, apparatus, product, or process disclosed, or represents that its use would not infringe privately owned rights. Reference herein to any specific commercial product, process, or service by trade name, trademark, manufacturer, or otherwise does not necessarily constitute or imply its endorsement, recommendation, or favoring by the United States Government of any agency thereof. The views and opinions of authors expresed berein do not necessarily state or reflect those of the United States Government or any agency thereof. 


\begin{abstract}
Central to our effort was the investigation of muon catalysis of nuclear fusion, in particular of muon lose processes and muon production. During a brief period in 1989/90 considerable effort had been devoted towards the investigation of Jones' report of nuclear fusion occurring in electrolysis of heavy water, with particular emphasis being the search for consistency with current knowledge of nuclear physics. This has stimulated an exploration into the catalysis of fusion by a yet undiscovered, stable, ultraheavy elementary particle. Our muon catalyzed fusion results are greatly encouraging in that we consider muon catalysed fusion in derse and degenerate plasma environments as having practical promise.
\end{abstract}

\title{
TABLE OF CONTENT
}

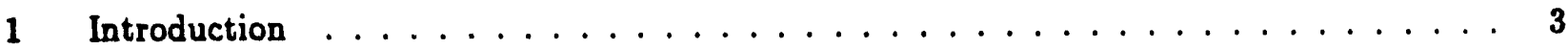

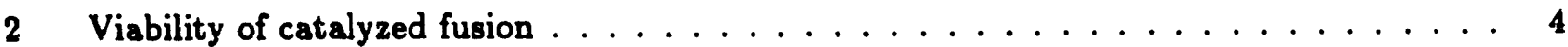

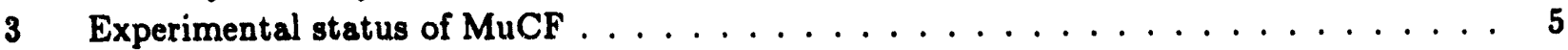

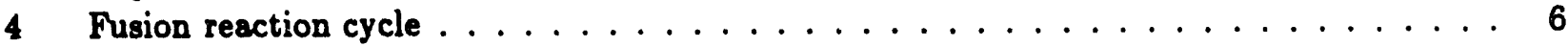

5 Muon sticking after fusion $\ldots \ldots \ldots \ldots \ldots \ldots \ldots$

5.1 Initial sticking after fusion $\ldots \ldots \ldots \ldots \ldots \ldots \ldots$

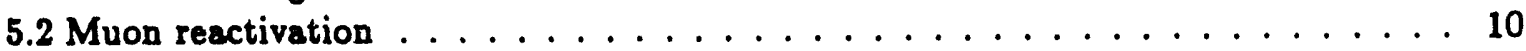

5.3 Density dependence of sticking? . . . . . . . . . . . . . . . 14

6 Evolution of muon amplitude after fusion $\ldots \ldots \ldots \ldots \ldots \ldots$

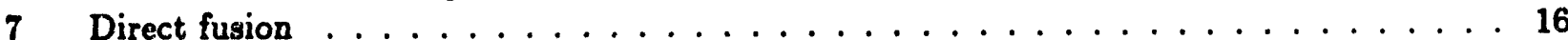

8 High density catalyzed fusion $\ldots \ldots \ldots \ldots \ldots \ldots$

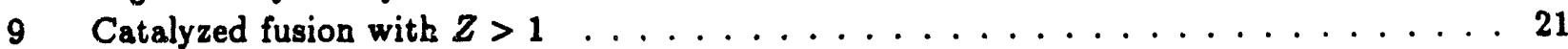

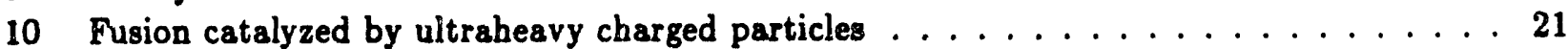

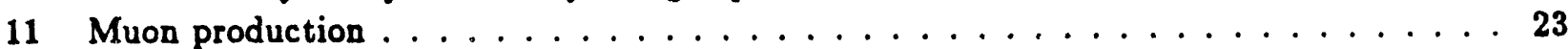

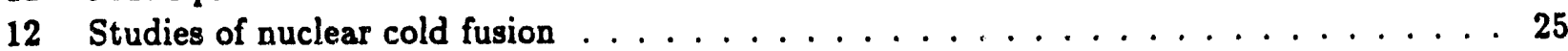

13 Principal publications $\ldots \ldots \ldots \ldots \ldots \ldots$ 


\section{Introduction}

Rather than to present the evolutionary steps which have lead to the present research position, and which are largely reflected in the publications completed under the auspices of the program (see Section 13), we shall primarily summarize here our current research position, indicating where appropriate what could be done in the future to clarify issues which were opened up by our progress. Following on the discussion of the viability of catalyzed fusion, we will present along with the key experimental results (Section 3) a short account of the physics surrounding the subject (Section 4). This is followed by a discussion in different sections of key research topics we have addressed. In consequence of the progress made, we believe that the feasibility of a small-scale fusion based on catalyzed reactions rests on either the remote chance that a yet undiscovered ultraheavy negatively charged elementary particle exists in Nature, or on the possible technical realization of a system based on muon catalyzed fusion (MuCF) in high density degenerate hydrogen plasma (density 1000 LHD, temperature $\mathrm{O}(100 \mathrm{eV}))$.

In Table 1 we show all hydrogen based fusion reactions. It was the objective of this research program to identify alternate paths to fusion such that the need for the high temperature plasma could be avoided. The fundamental idea is to use a negatively charged elementary particle to screen the Coulomb repulsion between positively charged hydrogen isotopes such that spontaneous fusion reaction can occur. Following a fusion reaction the negatively charged catalyst must be set free in order to facilitace a cycle of atomic and molecular processes which lead to a long chain of catalyzed nuclear reactions. This ides was indeed put forward independently by several researchers in the late forties: Frank hypothesized [1] that such catalyzed reactions could be at the origin of the strange events seen in cosmic ray data, and (as is reported) about the same time similar ideas were developed in the context of hydrogen bomb development in the Soviet Union by Zakharov. The catalyzed pd fusion process proposed by Frank in 1947 has been accidentally experimentally discovered 10 years later by Alvarez [2]: muons when stopped in hydrogen bubble chamber lead to occasional fusion events, whose signature was visually detected as an aberration in the muon decay characteristics.

Muons are unstable $(\tau=2.2 \mu s)$ radioactive particles, found in Nature as secondaries of the cosmic activity, and which need to be produced in elementary collisions in order to generate a usable abundance. The key property of muons which allows them to perform the catalysis is their relatively high mass (207 times that of electrons) which helps to localize their quantum mechanical probability distribution such that their charge is capable to reduce the repulsion between two light nuclei, permitting spontaneous fusion to occur at a useful rate. Muons are not unique in their ability to catalyze fusion: in principle all negatively charged heavy elementary particles are to be considered. Among the known, unstable elementary particles, muons have an exceptionally long life span which allows for many fusion reactions in conditions of "normal" density and temperature. Other today known elementary particles either decay too fast or/and have a preference to undergo a reaction with one of the fusing nuclei. There remains the possibility of a yet undiscovered elementary particle. Only an ultra heavy particle $r$ with $\mathrm{MX}_{\mathrm{X}}>10^{16} \mathrm{eV}$ could have escaped discovery today and, as our work shows (Section 10), such particles would be quite useful in catalyzing fusion. 
Table 1: Hydrogen fusion reactions

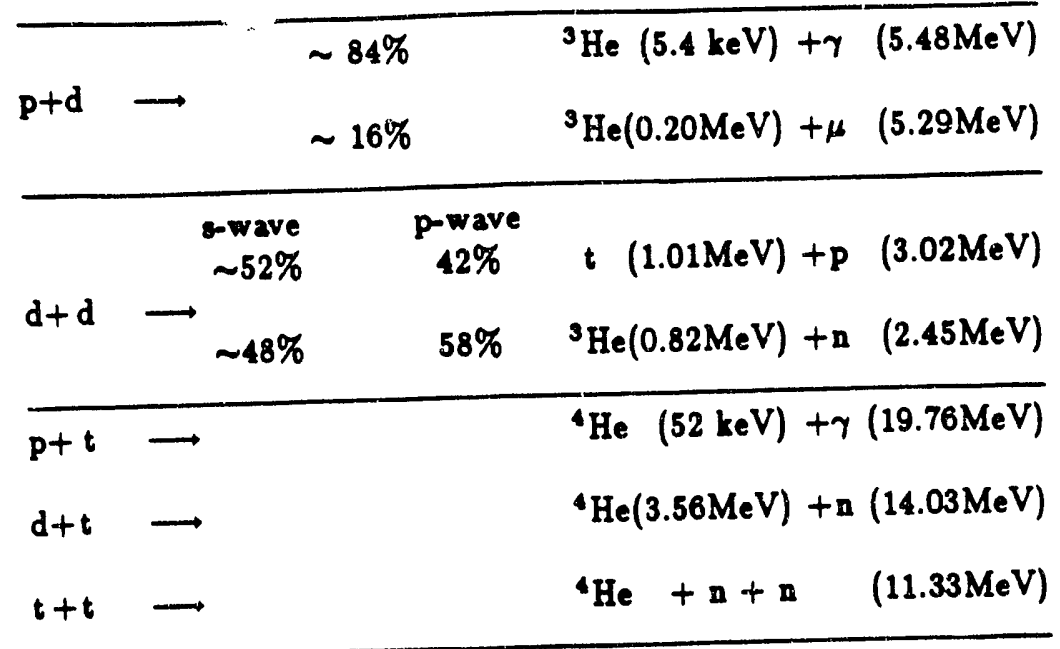

\section{Viability of catalyzed fusion}

In all approaches to fusion one seeks to understand the "break-even point", where the energy required to sustain fusion is exceeded by the fusion energy yield. In catalyzed fusion (CF) such a condition arises from comparison of the energy cost of the catalyst, presently a negatively charged muon, and this has to be compared to the fusion yield reachable before the catalyst is "poisoned" or simply decays due to a finite life time. We must consider the total rate of muon loss: in addition to its natural decay, muons can be captured by elements with $Z>1$, in particular also directly following the fusion reaction in the process called muon sticking to the helium nucleus, see Section 5 .

For details of atomic and molecular processes in MuCF we refer the reader to our recent review [3] in Advances in Atomic, Molecular and Optical Physics, Vol29. We note here that the achievable number of fusions $Y$ (yield) per muon, is obtained from the ratio of the cycling rate $\lambda_{c}$ (see Section 4 ) to the rate of muon loss $\lambda_{l}$. The latter contains, apart from the rate of muon decay $\lambda_{0}$, also the rate of muon loss due to sticking, $\lambda_{e} \omega_{s}^{d t}$. We have for the yield

$$
Y=\frac{\lambda_{c}}{\lambda_{l}}=\frac{1}{\lambda_{0} / \lambda_{c}+\omega_{0}^{d t}+\delta W_{l}}<W_{0}^{-1}<\frac{1}{\omega_{0}^{d s}}
$$

Best confirmed values today are $Y=150$ and $\lambda_{c}=10^{8} \mathrm{~s}^{-1}$. We note that it is not the rate of the nuclear fusion reaction $\lambda_{f}$, especially for the very energetic reaction $d t \rightarrow a n$ which limits the number of fusions per muon, but rather it is the rate of the different atomic and molecular processes accompanying a fusion cycle, which are also contained in $\lambda_{c}<<\lambda_{f}$. We note that:

$$
\lambda_{c}^{-1}=\sum \lambda_{i}^{-1}
$$

where the sum includes the rate of fusion as well as all the necessary rates governing the cycle of reactions. Figure 2 below gives a brief impression of the numerous rates involved, and we discuss the different processes required in the molecular cycle in Section 4.

Given the fusion yield, the $Q$-value of the fusion reaction fixes the energy yield:

$$
E_{Y}=Q Y \geq E_{\mu}
$$


which must be greater than the energy cost of muon production, $E_{\mu}$. This break-even condition is very different from the one known in hot fusion - in the latter case the energy loss through radiation is compared to rate of energy production. In muon catalyzed fusion (MuCF) we compare the characteristic nature of the catalyst to the fusion yield. In consequence, there is little dependence in MuCF on scale of size, and bence the magnitude of any final technical device solely powered by MuCF is too a large extend left open to economical optimization. Let us now sum up the current energetics of the muon catalyzed $d t$-reaction. Every $d-t$ fusion releases $17.6 \mathrm{MeV}$ and hence the maximal energy yield per muon is presently $2.7 \mathrm{GeV}$ stemming from a record yield of about 150 fusions. Thus the so called scientific break-even has been exceeded; that is, the amount of fusion energy release by a single muon during its catalytic cycle exceeds the minimal energy required to make the muon, which is $2 \mathrm{~m}_{\mu} \mathrm{c}^{2}$. As we shell discuss in Section 11 the cost of muon production is about $10 \mathrm{GeV}$ of thermal power. We are thus just about a factor 4 away from the engineering breakeven, the point at which a self contained reactor could perpetually operate without energy supply from outside. Economical breakeven may be another factor 5 - 10 away.

How can we gain a factor 20 or so required to make MuCF a fusion alternative? We take the position that in principle a much greater number of fusions per muon is possible, than $\simeq 150$ already achieved. A limit to the fusion yield per muon arises always in a particular context envisaged for the likely MuCF fusion apparatus. We believe that for high density degenerate plasma, the number of achievable fusions per muon is very large and that such an environment holds promise for practical applications of MuCF, see Section 8.

\section{Experimental status of MuCF}

The important measurement involves the determination of the fusion yield, or its inverse, which after some corrections is the probability of muon sticking in fusion $w_{0}$. For $d t$ fusion a surprisingly small value was discovered by Jones et al (1986) [4] at LAMPF and since confirmed at many laboratories including the PSI (Paul Scherer Institute formerly SIN), Triumph, KEK (Japan) and RAL. Most of the US-LAMPF dt-fusion sticking fraction results were obtained considering the cycling rate of muons and measuring the rate of muon loss. Similar experiments have now been repeated with a somewhat different analysis of kinetics of the neutron emission and cycle dynamics. An interesting novelty was the work of Hartmann [5] on the X-ray emissions from the MuCF cycle from which the sticking probability can be extracted by a theoretical analysis. In a tritium rich environment this is a very difficult experiment, as the natural triton decay generates a background just in the energy window of interest.

A summary of the experimental measurements of $\omega_{0}^{0}$ is given in Table 2 and Figure 1. While there is only a slight density dependence arising from the regeneration phenomenon in the theoretical calculations using pure three body Coulomb theory, there is a pronounced trend (within the error bars) for a decrease of sticking with density in the LAMPF data, which are just barely in agreement with the PSI - neutron based sticking data. However, the density dependence in the LAMPF data could also be related to the fact that the high density points were obtained by changing the temperature near or in the liquid DT-phase. It is interesting to note that these results are nearly half as small as the theoretical expectations. There is no doubt, in qualitative terms, about the significantly smaller experimental sticking than the theoretical expectation, considering the enormous neutron yield reported per muon, which can be as high as 150 neutrons [4]. Interestingly, $\mathrm{X}$-ray based sticking is in good agreement with the LAMPF neutron based data. The result shown in Table 2 was extracted using the theoretical yield of $K_{\alpha}$-X-rays per stuck muon: $K_{\alpha} / \omega_{0}=0.53$. 
Table 2: The $(\alpha \mu)^{+}$sticking fraction for different $d-t$ target densities $\phi=\rho / \rho_{0}$

\begin{tabular}{lcc}
\hline DENSITY $\rho=\phi \rho_{0}$ & $\phi=1.2$ & $\phi=0.1$ \\
\hline EXPERIMENT(from neutron detection) & & \\
\hline Jones et al. (1986) $[4]$ & $0.35 \pm 0.07$ & $1.1 \pm 0.5$ \\
Breunlich et al. (1987) $[6]$ & $0.45 \pm 0.05$ & $0.50 \pm 0.10$ \\
Nagamine (1987) $(7)$ & $0.42 \pm 0.07$ & \\
Bossy et al. (1987) $(8)$ & $0.42 \pm 0.14$ & \\
\hline
\end{tabular}

EXPERIMENT (from X-ray detection $K_{a} / \omega_{0}=0.53$ )

\begin{tabular}{llll}
\hline Hartmann (1990) $[5]$ & $0.36 \pm 0.09$ & \\
Nagamine (1990) $[27]$ & $0.38 \pm 0.11$ & \\
\hline THEORY using $\omega_{0}^{0}=0.915$ & & \\
\hline after regeneration & 0.59 & 0.65 \\
\hline
\end{tabular}

\section{Fusion reaction cycle}

Each catalytic cycle contains a number of processes in which with some small branching the muon can be delayed and/or neutralized. Therefore it is necessary to understand the fusion cycle history, including small probability alternatives. We will here briefly describe the complexity of the reactions to be considered in a ultra pure mixture of Deuterium and Tritium. The main steps of the dt nuclear cycle as shown in Figure 2 are summarized below, beginning with a free muon:

1. The $\mu^{-}$is slowed in the mixture of hydrogen isotopes within less than $10^{-10} \mathrm{~s}$ to atomic velocities, as can be deduced from muonic stopping power at LHD.

2. The $\mu$ is captured by one of the hydrogen isotopes $d$ or $t$ in proportion to their abundance $C_{d}, C_{t}$ in a high orbit $(n \approx 14)$, primarily by Auger processes.

3. Following the capture in an outer orbit:

a) If the muon is captured by a deuteron, it undergoes transfer to the beavier tritium isotope. From the $d \mu_{1}$-state transfer must compete with the $d d \mu$ formation rate or direct fusion.

b) If the muon is captured by tritium, the muon cascades down to the $(t \mu)_{1}$, state in $10^{-11} \mathrm{~s}$.

4. The $(t \mu)_{1}$, atom (with thermal or epithermal energy) collides with a $D_{2}$ or DT molecule and either it:

a) there is direct nuclear reaction from which the muon emerges generally free and returns to the beginning of the cycle.

b) or $(t \mu)_{1}$, forms (resonantly) the excited $(d t \mu)_{11}$, muomolecule embedded within the host electro-molecule. In this case in the muomolecule $d t \mu(11)$ an Auger transition follows, primarily to the $(J \nu)=(01)$ state. This occurs within $10^{-12} \mathrm{~s}$. Also within $10^{-12} \mathrm{~s}$ nuclear fusion takes place and the muon returns in most cases to the beginning of the cycle. 


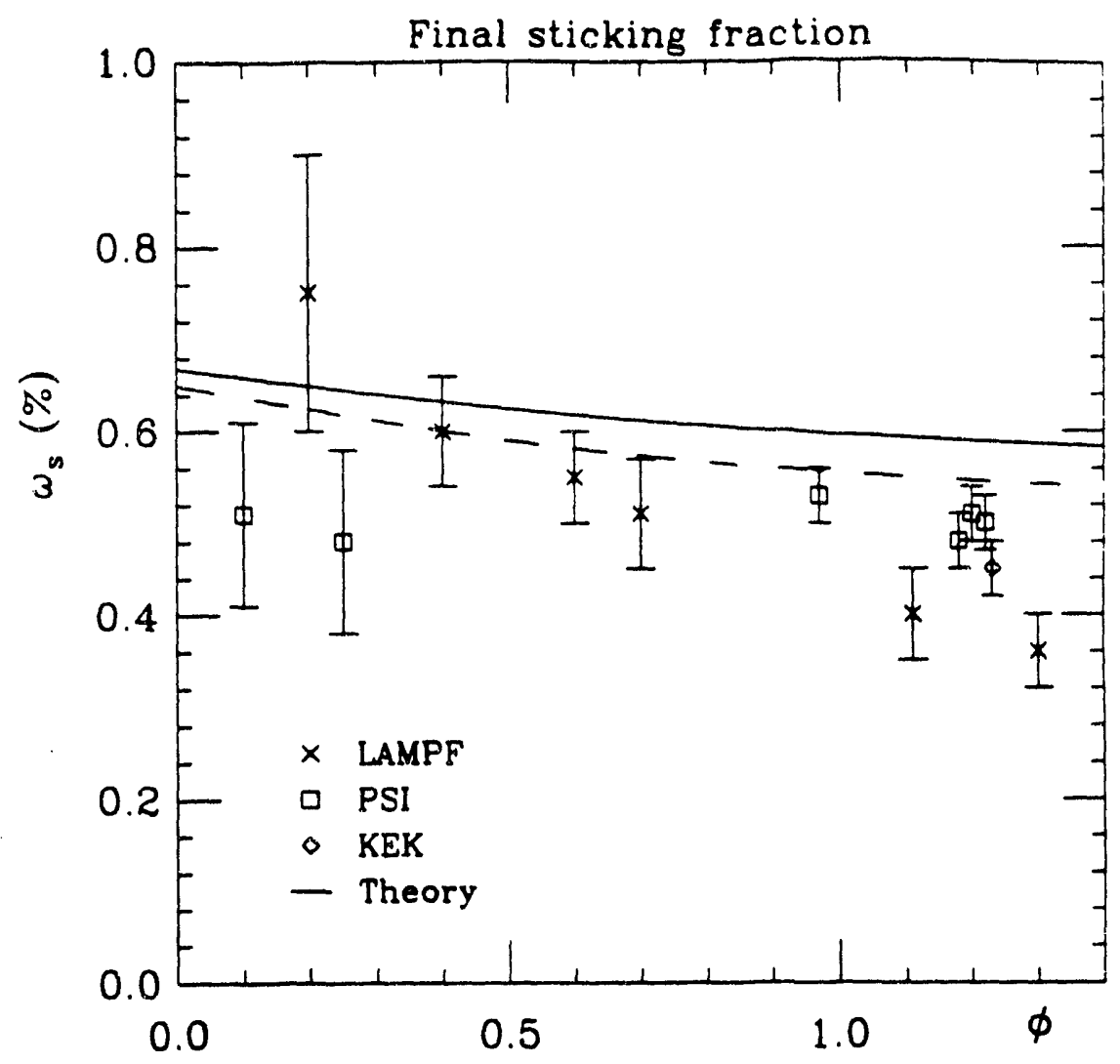

Figure 1: Experimental and theoretical final sticking fraction as a function of density.

Irrespective of the fusion process (direct fusion or intra- molecular fusion process) the muon is captured by (bound to) the product $\alpha$-particle in a small fraction of all reactions. The branching ratio between sticking and not sticking reactions is the initial sticking fraction, which may be reduced in the subsequent slow down process of the $\alpha \mu^{+}$ion, which carries about $1 / 5$ of the fusion energy yield (3.6 MeV). The molecular fusion cycle dominates at standard temperature and pressure conditions and is best studied today both theoretically and experimentally. At STP the muon stripping (regeneration reaches $30 \%$, see Section 5.2. In order to increase the regeneration probability it is necessary to consider degenerate plasms environments, in which theoretical calculations suggest that direct fusion processes dominate, see Section 8.

The population probability of the deuterium ground state $q_{1}$, plays an important role in muon cycle dynamice and a significant fraction of the average dt MuCF cycle time is taken up by the time the muon spends in this state; also it may lead to the dd fusion reaction. The experimentally observed (molecular cycle dominated) sticking fraction for $d t$ fusion is $0.45 \%$, which may be compared to $12.2 \%$ sticking probability for the $d d$ fusion branch reacting into the ${ }^{3} \mathrm{He}$-channel. At STP the molecular cycle dominates since there is a pronounced resonant molecular formation when a neutral $t \mu$ muostom enters a hydrogen molecule containing a deuteron. $t \mu$ can bind extremely weakly to a deuteron, the muomolecular binding energy being picked up by the (second) vibrational band of the host electro-molecule. The rate of formation of such resonances is highly temperature dependent. The understanding of this rate requires precise knowledge of the (11) state energy.

Because of the presence of the resonant process at STP, the molecular cycle dominates. However the nuclear fusion of the $d$ and $t$ occurs almost exclusively from the relative $J=0$ states, and 


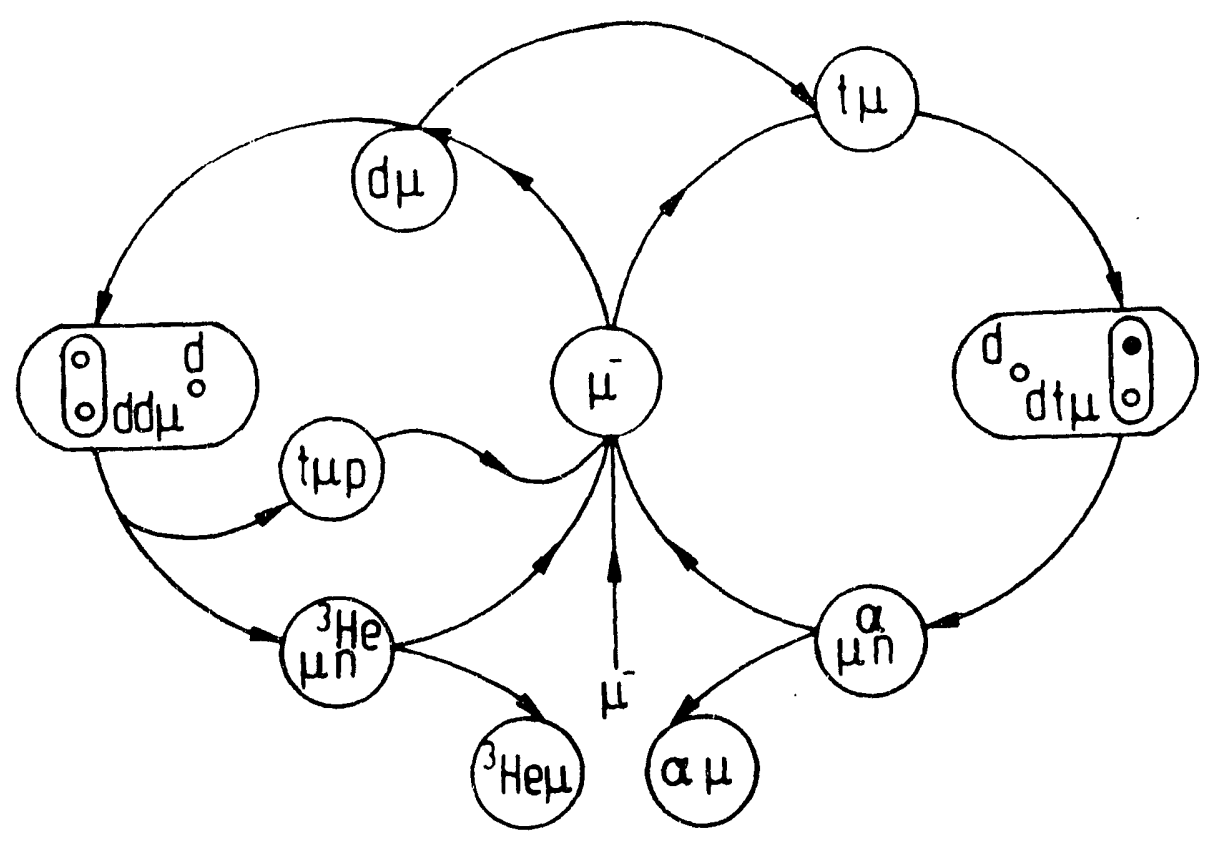

Figure 2: Representation of $d-t-\mu$ fusion cycle processes

hence an Auger cascade is needed to prepare the initial state. The fusion proceeds also practically exclusively via a ${ }^{5} \mathrm{He}\left(\frac{3}{2}^{+}\right)$nuclear resonance located $50 \mathrm{keV}$ above the $d+t$ threshold. The fusion rate has been computed from the ground state and first vibrational molecular state to be of the order of $10^{12} \mathrm{~s}^{-1}$; the fusion rate from the (11) state is estimated to be of the order $10^{8} \mathrm{~s}^{-1}$, and is primarily due to the non-adiabatic components in the wavefunction.

Fusion in the $d d$ system is somewhat different due to the symmetry of the relative dd wave function. The transition rate from the $(d d \mu)(11)$ state can only take place if accompanied by a spin flip of one of the deuterons. The rate of this transition is sufficiently slow (37.3 \pm 1.5$) 10^{6} \mathrm{~s}^{-1}$ [9], for $P$-wave fusion to occur directly from the (11) state. The value of the branching ratio found in the muon catalyzed fusion reactions at $\mathrm{T}=293 \mathrm{~K}$ is $1.4 \mathrm{in}$ favor of ${ }^{3} \mathrm{He}$ production. Most interesting is the result [9] that this branching ratio drops below unity in MuCF d-d reactions at $T=70 \mathrm{~K}$. At this temperature the resonant molecular formation rate has decreased enough and other mechanisms leading to the $d$-d fusion, e.g. Auger molecular formation or direct fusion resction dominate the $d d$ fusion cycle.

The role of resonant processes in MuCF can not be understated: the extremely weakly bound muomolecular states in the $d d \mu$ and $d t \mu$ molecules facilitate the formation of muomolecules and hence these two cycles are considerably faster than other catalysis cycles, and known to have a strong temperature dependance. The main reasons for the particular attention given to the dit case are:

1. The $d t \mu$ system possesses a $(J \nu)=(11)$ molecular state believed to be bound by just $596 \pm$ $2 \mathrm{meV}$, which can resonate with second vibrational band of the $D_{2}$ molecule. 
2. The $d t$ nuclear system has a $\left(\mathrm{J}^{x}=\frac{3}{2}^{+}\right)$nuclear resonance just $50 \mathrm{keV}$ above the $d+t$ threshold, with a total width of $70 \mathrm{keV}$ and hence the nuclear $d-t$ reaction strength is 100 times faster than any other involving hydrogen isotopes;

3. The $Q$-value for the $d+t \rightarrow \alpha+n$ reaction is $17.60 \mathrm{MeV}$, making it one of the most energetic of all bydrogen-hydrogen reactions.

The last property is important directly and indirectly for energy yield per muon: the high postfusion velocity $v_{\alpha-n}$ is at the origin of the small muon loss to the produced $\alpha$-particle, and also it is the source of the possible muon regeneration.

\section{Muon sticking after fusion}

\subsection{Initial sticking after fusion}

The probability of the initial sticking is the resction branching ratio, in the case of the $d t$-fusion:

$$
\omega_{s}^{0}=\frac{\Gamma(d t \mu \rightarrow n+\alpha \mu)}{\Gamma(d t \mu \rightarrow n+\alpha+\mu)+\Gamma(d t \mu \rightarrow n+\alpha \mu)},
$$

which is small, but significant in the context of possible MuCF applications. The total initial sticking probability $\omega_{0}^{0}$ is actually the sum of the probabilities with which the muon sticks in any $\alpha$-particle bound state directly after fusion:

$$
\omega_{1}^{0}=\sum_{n d} \omega_{n d}^{0} .
$$

Assuming that the nuclear interaction can be accounted for as a perturbation, the branching ratio of the reaction is found to be:

$$
\omega_{a}^{0}=\frac{\sum_{n d}\left|\left\langle n|| V_{\text {nuc }} \mid i\right\rangle\right|^{2}}{\sum_{n d}\left|\left\langle n l\left|V_{\text {nuc }}\right| i\right\rangle\right|^{2}+\sum_{c}\left|\left\langle c\left|V_{\text {nuc }}\right| i\right\rangle\right|^{2}}
$$

where $|n l\rangle$ are the final states with a stuck muon and $|c\rangle$ those with a free continuum muon, and $V_{\text {nuc }}$ is the nuclear interaction. All bra- and ket- states in Eq. (6) are solutions of the Coulomb problem; hoswever for different initial and final state Hamiltonians. It can be shown (see Reference [10], Section 3.1 and references therein) that Eq. (6) is accurate and does not invoke the so called sudden approximation, as long as it is possible to ignore the action of the nuclear interaction. In that case sticking is:

$$
\omega_{n d}^{0}=\left|\int d^{3} r \varphi_{n d}^{0}(r) e^{-i k r} \frac{\psi(r, 0)}{\left(\int d^{3} r|\psi(r, 0)|^{2}\right)^{1 / 2}}\right|^{2} .
$$

With the adiabatic approximation to the three-body wave function:

$$
\psi(r, R)=\psi \operatorname{rc}(r ; R) \chi(R)
$$

where:

1. the two center (TC) amplitude of the muon refers to a solution for a frozen position of the hydrogens at separation $R_{\text {; }}$

2. the relative nuclear motion amplitude $\chi(R)$ describes nuclear vibrations under the influence of the mutual Coulomb repulsion of the nuclei, kept together by the molecular potential $V_{q m}=E(R)$; 
3. $E(R)$ is the Coulomb eigen energy to which at a given separation $R$ the amplitude $\psi_{T C}$ is an eigenstate.

Inserting Eq. (8) into Eq. (7) one finds the classic expression first obtained by Jackson [11]:

$$
\omega_{n d}^{a d}=\left|\int d^{3} r \varphi_{n d}^{*}(r) e^{-i \mathbf{k} \cdot r} \psi_{T C}(r ; 0)\right|^{2}
$$

Here $\psi_{T C}(r ; 0)$ is the muon wave function computed for the combined nuclei, i.e. the muonic ${ }^{5} \mathrm{He}-1 \mathrm{~s}$ eigenstate.

Note that in these early discussions of the sticking process, it was customary to forget that the muon is described in terms of the time dependent $\alpha$-co-moving state. This omission led to claims for the so called sudden spproximation, i.e. that the sticking occurs instantly after fusion, in which case it is not necessary to consider the time evolution of the muon amplitude. One of our early contributions [12] was to clean up this confusion: the expression we presented above are the true $t \rightarrow \infty$ amplitudes for sticking (adiabatic as well as nonadiabatic). From this observation arises the necessity to study the possibility that the final sticking fusion amplitude takes so much time to form, that its coherence could be disrupted by interactions in dense matter. We will return to this issue in Section 6. We note here that in order to be able to describe this phenomenon we developed a Monte Carlo Wigner-Function approach to the evolution of the fusion amplitude $[13,14,15]$. How and if these considerations affect sticking remains at present unresolved.

The adiabatic sticking probability is $\omega_{0}^{0} \sim 1.2 \%$ as shown in the first column of Table 3 . More sophisticated three-body non-adiabatic wave functions $[16,17,18,19]$ lead to a somewhat smaller value of $\omega_{0}^{0}=0.89 \%$, which is slightly dependent on the initial molecular state. The non-adiabatic results presented in Table 3 are for the $(n l)=(01)$ state from which most of the nuclear reactions from within th nolecular system occur. In the last column of Table 3 the initial sticking fractions into the $\mathrm{rl}$ staten are presented as obtained allowing for the R-matrix perturbative nuclear impact on the muon fusion amplitude. $\omega_{\theta}^{0}=0.92 \%$ is today viewed by the majority of MuCF researchers as the best value for the total initial sticking probability, questioned only in the context of possible non-perturbative nuclear phenomena.

\subsection{Muon reactivation}

Our own work has addressed in considerable detail the issues surrounding muon regeneration. Once the muon sticks to the $\alpha$-particle, it is not entirely lost from the cycle of reactions: at the initial velocity of about $v_{\alpha \mu}=5.82 \alpha c$; it carries about $86 \mathrm{keV}$ kinetic energy, which is significantly greater than the energy needed, $11 \mathrm{keV}$, to strip it from the $\alpha$-particle. Even more importantly, it takes many atomic collisions before the $\alpha^{+}{ }^{+}$-ion looses its energy, ca. $3.5 \mathrm{MeV}$. In order to relate the

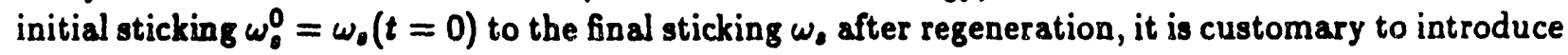
$R$, the reactivation probability, which can be density dependent. The finsl sticking is:

$$
\omega_{0}=\omega_{0}^{0}(1-R) \text {. }
$$

The diverse muon stripping processes compete with the rate of energy loss of the $(\alpha \mu)^{+}$-ion in the hydrogen medium, which depends upon the stopping power $S(v)$ and density $\rho$ of the hydrogen:

$$
\frac{d E}{d t}=-\rho v S(v)
$$


Table 3: Partial sticking probabilities $\omega_{n d}(\%)$ for the (01) state for the reaction $(d t \mu) \rightarrow\left(\mu^{4} \mathrm{He}\right)+n$.

\begin{tabular}{cccc}
\hline$n l$ & $\omega_{n l}^{0}(\mathrm{BO})^{a}$ & $\omega_{n l}^{0}(01)^{b}$ & $\omega_{n l}^{0}(01)^{c}$ \\
\hline $1 \mathrm{~s}$ & 0.9030 & 0.6840 & 0.71 \\
$2 \mathrm{~s}$ & 0.1287 & 0.0981 & 0.10 \\
$2 \mathrm{p}$ & 0.0321 & 0.0240 & 0.02 \\
3 & 0.0509 & 0.0386 & 0.04 \\
4 & 0.0220 & 0.0167 & 0.02 \\
5 & & 0.0086 & 0.01 \\
all others & & 0.0181 & 0.02 \\
\hline total & 1.1645 & 0.8881 & 0.92 \\
\hline
\end{tabular}

- Sticking fractions in Born-Oppenheimer approximation [11].

Coulomb aticking fractione from References $[18,19]$.

'Sticking fractions including nuclear interaction modifications of the wave function $[20,21]$.

The time required to bring the $(\alpha \mu)^{+}$-ion to rest in liquid hydrogen is of the order of $t_{\text {stop }} \approx 4 \times 10^{-11}$ at LHD, so muon stripping, if it occurs, does not have any impact oin the cycling rate of the muon. In the simplest first estimate of the significance of regeneration let us assume that all muons captured in excited states rapidly fall into the 1s-state, which is thus populated with the initial probability $\omega_{\mathrm{g}}^{0}$. Allowing only for one step processes we find:

$$
\frac{d \omega_{0}}{d t}=-\lambda_{\text {otr }}(v) \omega_{0},
$$

where $\lambda_{\text {str }}(v)=\sigma_{\text {otr }}(v) \rho v$ is the rate of muon stripping from the ground state, with the stripping cross section $\sigma_{\text {str }}(v)$ (sum of ionization and transfer cross sections). Using the energy rather than the slowdown time of the $\alpha$-particle as the integration parameter and combining Eq. (11) and Eq. (12), the stripping fraction for the ground state is:

$$
1-R_{0}^{f}=\exp \left(-\int_{E_{f}}^{E_{0}} \frac{\sigma_{\text {otr }}(E)}{S(E)} d E\right),
$$

where $E_{0}$ is the initial and $E_{f}$ the final energy of the $(\alpha \mu)^{+}-$ion. The stripping fraction is therefore exponentially the ratio of the muon stripping cross section to the energy loss weighted electron ionization cross section referred to generally as the stopping power $S$. In this simple calculation, $\mathrm{R}_{0}^{f}$ for $E_{f}=0$ is found to be about 0.3 .

In a complete treatment of the muon reactivation process necessary for example to obtain the density dependence or the $\mathrm{X}$-ray yield not only the 18 state, but also all excited states must be included in the population equations, each of which has different initial amplitudes, see Table 3 and different stripping cross sections. The excited state populations are coupled by radiative, Auger and Coulomb induced de-excitation and Stark mixing (quenching) processes. The reactivation probability $\mathbf{R}$ of the muon after fusion can be calculated including all above processes by solving the following coupled differential equations numerically:

$$
\begin{gathered}
\frac{d P_{i}}{d t}=-\lambda_{i o n}^{i \rightarrow \infty} P_{i}-\sum_{m} \lambda_{t r a}^{i \rightarrow m} P_{i} \\
-\sum_{k>i} \lambda_{e s c}^{i \rightarrow k} P_{i}+\sum_{k<i} \lambda_{e x c}^{k-i} P_{k}+\sum_{k>i} \lambda_{A u}^{k \rightarrow i} P_{k}
\end{gathered}
$$


Table 4: Reactivation coefficients of the $(\alpha \mu)^{+}$reaction $R$ as a function of density with quenching cross section $\sigma_{2 \gamma \rightarrow 2 p}=0.0037 \mathrm{a}_{0}^{2} v^{-2}$ and without quenching $(Q=0)$ [23].

\begin{tabular}{ccc}
\hline density & $R$ & $R(Q=0)$ \\
\hline 0.0 & .2782 & .2782 \\
0.2 & .2908 & .3181 \\
0.4 & .3093 & .3441 \\
0.6 & .3251 & .3653 \\
0.8 & .3376 & .3815 \\
1.0 & .3478 & .3938 \\
1.2 & .3561 & .4036 \\
1.4 & .3633 & .4115 \\
1.6 & .3698 & .4183 \\
1.8 & .3752 & .4239 \\
2.0 & .3802 & .4290 \\
\hline & & \\
& & \\
$-\sum_{k<i} \lambda_{\text {rad }}^{i \rightarrow k} P_{i}+\sum_{k>i} \lambda_{\text {rad }}^{k \rightarrow i} P_{k}$
\end{tabular}

and

$$
\frac{d F_{i}}{H_{i}}=\sum_{i} \lambda_{s t r}^{i} P_{i}
$$

with

$$
\lambda_{\text {otr }}^{(i)}=\lambda_{\text {ion }}^{i \rightarrow \infty}+\sum_{m} \lambda_{\text {tra }}^{i \rightarrow m}
$$

$P_{i}(t)$ is the population of the $(\alpha \mu)^{+}$-ion states as a function of time $t, P_{0}$ is the number of stripped muons. The reactivation probability or reactivation probability $R$ is:

$$
R=\int_{0}^{t_{\text {otopos }}} \sum_{i} \lambda_{\text {otr }}^{i} P_{i}(t) d t
$$

The multiple step processes involving radiative transitions are the source of a weak density dependence in the reactivatiou probability $[22,23]$, see Table 4 . One finds in the complete analysis that Eq. (13) is correct in the limit of zero density, in which case the transitions towards the $(\alpha \mu)^{+}$ ground state dominate all intermediate excitation processes.

There are a priori two paths to enhance regeneration. One possibility is to identify external conditions in which the stopping power is reduced - as alluded to this is the case in degenerate plasmas [24]. Indeed, it seems that regeneration can be nearly complete in such environment. Another possibility is to re-accelerate the $\alpha \mu^{+}$-ion, which has been considered in some detail by Kulsrud [25], and it requires an essentially matter free space, which is, however, inconsistent with the assumption that $\alpha \mu^{+}$-ions are generated by fusion processes in a dense hydrogen target.

There seems to remain a discrepancy between the here presented values of final sticking and the experimental results. Therefore we have considered the alternate measurement of sticking by observation of the X-ray yield. Should the muon be bound at any time to the $\alpha$-particle, there will be some muonic X-ray transitions. These arise either from the initial population of the excited states or from the excitation of the ground state during the $\alpha$-particle slowdown process. In either 
Table 5: Number of X-rays per $100 d$-t-fusions: $X Y_{K_{a}} \times 100$ for densities $\phi=\rho / \rho_{0}$.

\begin{tabular}{|c|c|c|c|c|}
\hline & $X Y_{K_{a}}$ & $X Y_{K_{0}}$ & $X Y K_{p} / K_{\alpha}$ & $X Y K_{\gamma} / X Y K_{a}$ \\
\hline $\begin{array}{l}\text { EXPERIMENT: } \\
\text { Hartmann (1990) [5] } \\
\text { Nagamine (1990) [27] }\end{array}$ & $\begin{array}{c}\approx 1 \\
0.19 \pm 0.05 \\
0.20 \pm 0.06\end{array}$ & & $\begin{array}{l}\phi \approx 1 \\
\leq 0.08\end{array}$ & $\begin{array}{c}\phi \approx 1 \\
0.02 \pm 0.013\end{array}$ \\
\hline $\begin{array}{l}\text { THEORY: } \\
\text { Cohen (1988) [28] } \\
\text { Markushin (1988) [29] } \\
\text { Takahashi (1988) [30] } \\
\text { Rafeliski H E et al (1989) [23] } \\
\text { Stodden et al (1990) [26] }\end{array}$ & $\begin{array}{l}\phi=1.2 \\
0.26 \\
0.26 \\
0.25 \\
0.31 \\
0.31\end{array}$ & $\begin{array}{c}\phi=0.1 \\
0.31 \\
0.31 \\
\\
0.39 \\
0.36\end{array}$ & $\begin{array}{l}\phi=1.2 \\
0.12 \\
0.12 \\
0.18 \\
0.07 \\
0.082\end{array}$ & $\begin{array}{c}\phi=1.2 \\
0.019 \\
0.018 \\
0.024 \\
0.012 \\
0.021\end{array}$ \\
\hline
\end{tabular}

NOTE: Theoretical results aie re-normalized to $\omega_{0}^{0}=0.915 \%$.

case the radiative transitions occur in competition to the other density driven Coulombic processes and hence their observed intensity providis key supplementary informstion about sticking. The K-series X-ray yield per muon fusion, $K X_{i \rightarrow 1}$ is obtained from the population probability $P_{i}(t)$ of the $(\alpha \mu)^{+}$states, given the transition rates $\lambda_{\text {rad }}$ :

$$
K X_{i \rightarrow 1}=\int_{0}^{t \text { otop }} d t \lambda_{\text {rod }}^{i \rightarrow 1} P_{i}(t) \omega_{0}^{0} .
$$

A detailed investigation [23] of the dependence of the KX-yield on the diverse phenomena controlling the history of the $(\alpha \mu)^{+}$-ion has shown, that significant differences are only brought about through the choice of the Stark mixing in the L-shell: turning off the mixing reduces the $K_{\alpha} \mathbf{X}$-ray yields by $30 \%$. On the other hand, significant modifications of the stopping power could impact the KX-yields. In particular, a reduction of the stopping power, which leads to greater reactivation (and hance smaller final sticking), increases the yield of muonic X-rays emitted after fusion. A qualitative expression for $P_{i}[23]$ shows, that the $K_{a} X$-ray yield due to excitations into the L-shell is:

$$
K X_{\alpha} \sim \frac{\sigma_{\text {ese }}^{K \rightarrow L}}{S(v ; \phi)}\left(1+\frac{\lambda_{\text {eze }}^{L}}{\lambda_{\text {rad }}^{3 p}}\right)^{-1},
$$

where the ratio of the K-L excitation cross section to the stopping power enters. Consequently, a (density dependent) redurtion of the stopping power enhances the $K X_{\alpha}$ yield. This observation precludes any ad hoc manipulation of the stopping power of the $(\alpha \mu)^{+}$-ion with the goal of reducing the final sticking, as this leads to an enhancement of the theoretical X-ray yield.

Taking the beat theoretical sticking value of $0.92 \%$ to renormalize in prior theoretical results on X-ray yields accordance with Eq. (20), we arrive at the theoretical values shown in Table 5. It seems that the our theoretical results for $X Y_{K_{*}}$ [23], which were since confirmed by another study, [26] are a factor 1.5 too large compared to the experimental values $[5,27]$. This difference may be taken as a further suggestion that muons are captured by fusion $\alpha$-particles less often than expected. 


\subsection{Density dependence of sticking?}

There is a clear disagreement between predicted values of $\omega_{a}$ and the experimentally observed values at high density with which unusually high fusion yields (more than 150 neutrons per muon [4]) is associated. The source of this discrepancy could lie with any of the possibly misunderstood aspects of the catalyzed fusion cycle, which is density dependent. But any density dependence (beyond the trivial linear dependence of all two body rates) requires either a competition between a two body rate and a density independent rate, typically a radiative transition or the competitive presence of three body processes. A third alternative is to understand the density dependence as a temperature dependence in the range of $10-40 \mathrm{~K}$, since the high density points were obtained changing at the same time temperature and density. If the density dependence of sticking is to be explained, its understanding aught to be related to the following effectively density dependent processes known at present in the MuCF cycle:

1. the probability to reach the ground state of $d \mu$ (the 80 called $q_{1}$, probability). Here radiative $K_{a}, K_{\rho}$ transitions in $d \mu$ compete with excited state transfer rates $d \mu \rightarrow \mathfrak{L} \mu$;

2. the muon recapture probability (the convoy effect);

3. the probability of muon regeneration, where according to the present und anding only a small density dependence arise from the competition of 2p-1s transition with excitation into higher orbits;

4. there is a nonlinear density dependence in electro-muo-molecule resonance formation (three body effect) coupled with the possibility of a small sticking side cycle of MuCF.

The original estimates of $q_{1}$. predicted a strong density dependence. However, data $[31,4]$ appear to contradict this result showing $q_{1}$, to be only weakly density dependent and is in addition larger than theoretical values. $q_{1}$, is not properly understood, but in view of the experimental results it does not appear to be the source of the $w_{\text {s }}$ problem. The muon convoy effect would, even if it led to a resclution of the density dependence, leave us with a very small value of the initial sticking which requires a new phenomenon, perbaps related to non-perturbative interference of atomic and nuclear interaction. However, the preliminary results concerning the direct measurement of $\omega^{0}$ at very low density (the RAL-LAMPF experiment [32]) appears to rule this out, although the error bars are large at this preliminary stage; it is thus probable that the low density initial sticking is in agreement with conventional predictions. The final conventional source of density dependence may be some unaccounted for factor in the reactivation probability. Attempts have been made to explain this in terms of a density-dependent stopping power [23]. It is found (see Section 5.2) that a density-dependent reduction of the stopping power could explain the density dependence of the effective atinising; however, modification of the X-ray spectrum from transitions within the $(\mathrm{He} \mu)^{+}-$ion increzses the current discrepancy already present between theoretical predictions and experimental dat for $d d \mu$ and $d t \mu$ fusion.

\section{Evolution of muon amplitude after fusion}

While working on the problem of stripping (regeneration) of the muon from the $\alpha \mu$ during its slowing in passage through matter [12], we realized that we must also address the "oppositen phenomenon: some of the unbound muons will travel along with the $\alpha$ particle in the Coulomb cusp, and could be captured. The importance of this phenomenon would be that at the same time it allows to understand the density dependance of aticking [4], since muons cascading in flight from 
the high $\alpha$ orbit will often be stripped again.

As a first step we computed [34] the "capture" of the muon into the co-moving continuum states in the so called Coulomb cusp leading to enhancement of the muon phase space distribution in the vicinity of the outgoing $\alpha$-particle. Some of these so-called "convoy" muons will recombine with the a-particle due to interactions with the surrounding hydrogen target matter. This is possible since contrary to naive expectations, muons travelling with a velocity greater than that of the emerging helium nucleus slow down more rapidly traversing the target matter than the $\alpha$-particle despite their four times smaller stopping power, because of their smaller mass. Hence the $\alpha$-particle eventually catches up with muons that were initially running ahead, and these can then be captured into bound states by emission of radiation or by external Auger processes. We therefore also turned our attention to the problem of muon capture in flight [35]. Environmental conditions, e.g. target density will in principle influence the fraction of muons available to secondary capture.

The muon energy spectrum after $d+t$ fusion was obtained and compared to the case of $d+d$ fusion. Here the convoy effect was found to be less important, consistent with the possibility that the density dependant effects are mainly present in the $d+t$ case. We found that the fraction of the muon spectrum containing only muons traveling ahead of the $\alpha$-particle amounted to $0.83 \%$, compared with less than $0.1 \%$ if the Coulomb attraction of the $\alpha$-particle was switched off. In this work it was asouming that the final state muon amplitude can evolve coherently without interaction with the surrounding matter. This assumption is not necessarily correct - muons after fusion will in general undergo a number of complex processes during their slow down in matter - when they are bound to the $\alpha$-particle, or accompany it in a convoy cusp. During this time both otripping and recapture of convoy muons initially travelling in continuum states in respect to the muon can occur, in particular also the interaction with surrounding matter can contribute to incoherent processes. The recapture process adds to the initial sticking and may potentially lead to density dependent total sticking. Both sticking and convoy muons occur with a similar probability [34] of the order of $O(1 \%)$. In these studies an important problem is the determination of the muon capture rate into hydrogen or helium. Muon capture can proceed either by Auger transitions, by three-body collisions incorporating neighboring atoms or by radiative transitions. We have as a frst step, calculated the radiative capture cross sections from an initial continuum state to a final a final boundstates [35] for continuum states with kinetic energies of about $10 \mathrm{keV}$ and maximum energies up to the order of $100 \mathrm{keV}$.

We had thus embarked on a major numerical project with the aim of understanding the (incoherent and coherent) processes directly following the fusion reactions. Our key point with potentially significant impact on understanding of muon sticking is the recognition that even though the sticking amplitude in its magnitude is invariant in time, its phase space build up occurs over a relatively speaking very long time, while the $\alpha$-particle travels several $A$. We studied the evolution in the laboratory frame in time of the muon distribution function $f(\vec{r}, \vec{p})$ and we obtained a number of quantities of interest, such as muonic energy spectrum, distributions in position and momentum space suitably projected to illustrate the possibility of muons being recaptured by the $\alpha$-particle. Recognizing that the sticking probability evolves during an apprecisble distance along with the travelling $\alpha$-particle it must be kept in mind that there can be other interactions, such as Coulomb scattering from other matter acting on the muon during the time its post-fusion amplitude develops. Preliminary results of this program of research were reported (see [15] and references therein). However we have not yet determined if presence of matter and/or muon recapture processes impact the final sticking probability significantly. 


\section{Direct fusion}

Much of the detailed investigations of muon catalyzed fusion reactions of the past year has centered upon an investigation of the non-resonant in flight processes in $\mathrm{dt}$ fusion leading to the fusion of $d$ and $\mathrm{t}$. This involved the development of the $R$-matrix formulation for the $\mathrm{dt}$ system to incorporate the presence of the muon, and the details of this work are contained in the $\mathrm{Ph} . \mathrm{D}$. thesis of David Harley (unpublished). Two main processes have been examined; reactions in which the dt fuse above the $t \mu+d$ continuum without the formation of an ititermediate (11) $d t \mu$-molecular state, and transitions from above the $t \mu+d$ threshold to the non-resonant (quasiresonant) $d t$ amplitude existing just below threshold.

The in-flight fusion processes has been computed by obtaining the $d t$ nuclear waves in an effective potential generated by the muon. The $R$-matrix for the dt-an system was then used to match the $d t$ nuclear waves to the $\alpha$ waves, to obtain the fusion crose section. A rate of $2 \times 10^{5} \mathrm{~s}^{-1}$ was obtained at room temperatures [38]. Since the $d t-l=0,2$ waves are coupled to the $\alpha$ continuum, these waves extend as a continuum to below the $d+t \mu$ threshold. Thus transitions can be made to a fusing dt state at any energy above the an threshold. In the presence of the muon, these transitions are mainly expected to occur in the region of the Coulomb resonances, viz. the $(01)$ and $(00)$ states of the $d t \mu$ molecule, as the amplitude of the dt-an continuum state is greatly enhanced by the existence of Coulomb bound states in the $\mathrm{dt}$ region. However, a transition from above the $d+t \mu$ threshold to these Coulomb resonances are inhibited by the large energy that must be carried away by an Auger electron, and transitions to the below threshold are believed to be mediated by the formation of the intermediate (11) $d t \mu$ state. Even transitions to the (11) state are suppreased by the electron matrix element by the need to make a transition to the second vibrational state of the composite ((dt $\mu)$ dee) electro-molecule. This can be avoided if a transition is made from directly above the $d+t \mu$ threshold to the continuum $d t-\alpha n$ state directly below threshold, exciting just a rotational state in the $((d t \mu)$ dee) molecule. The electron matrix element is then of order unity; the suppression is now in the $d t$ matrix element, which is no longer resonant.

In Figure 3 we show the non-resonant continuum of nuclear states which exists both above and below the $d+(t \mu)_{1}$ e threshold (up to $-17.60 \mathrm{MeV}$ ). The continuum exists as a consequence of the above discussed coupling of the $d t \mu$ channel to the $\alpha n \mu$ continuum, and the stationary states may be easily constructed as the $R$-matrix is well known for this system. These global stationary continuum penetrates deep into the $d t$ region near to the 3-body Coulomb eigenenergies, with resonantly enhanced amplitude, and generally has an amplitude about $10^{-4}$ smaller away from these eigenenergies. Furthermore, just below (and above) the $d+(t \mu)_{1}$, threshold, the amplitude diverges weakly as the energy of the $d t \mu$ system approaches the $d+(t \mu)_{1}$, threshold. In order to be able to involve this near threshold continuum in the nuclear reaction processes, there must be other bodies to pick up the (small) surplus energy. Hence such reactions are of importance at high density.

In Figure 4 we show the impact of the direct pseudo-resonant rate on sticking, seen as function of temperature, assuming as a parameter the strength of the reaction. The consistency of data with this type of theory [33] suggest that the pseudo-rescnant direct fusion reaction mechanism could indeed be responsible for the smallness and vulnerability of observed sticking. It seems to us that it is necessary to perform a complete re-evaluation of all raw MuCF data with the explicit allowance for a direct reaction mechanism of varying strength. 


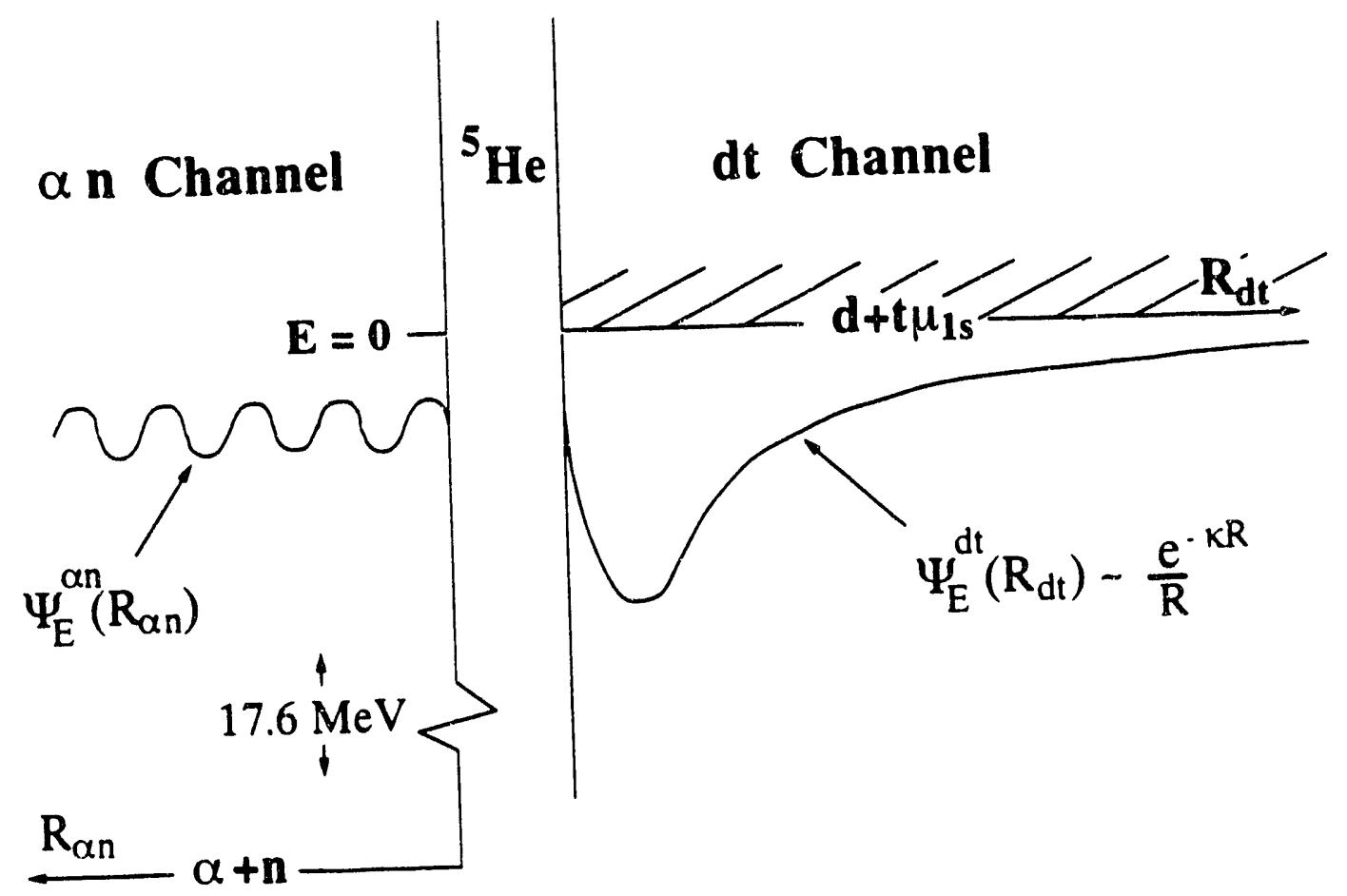

Figure 3: The $d t-\alpha n$ continuum just below the $d+(t \mu)_{1}$, threshold.

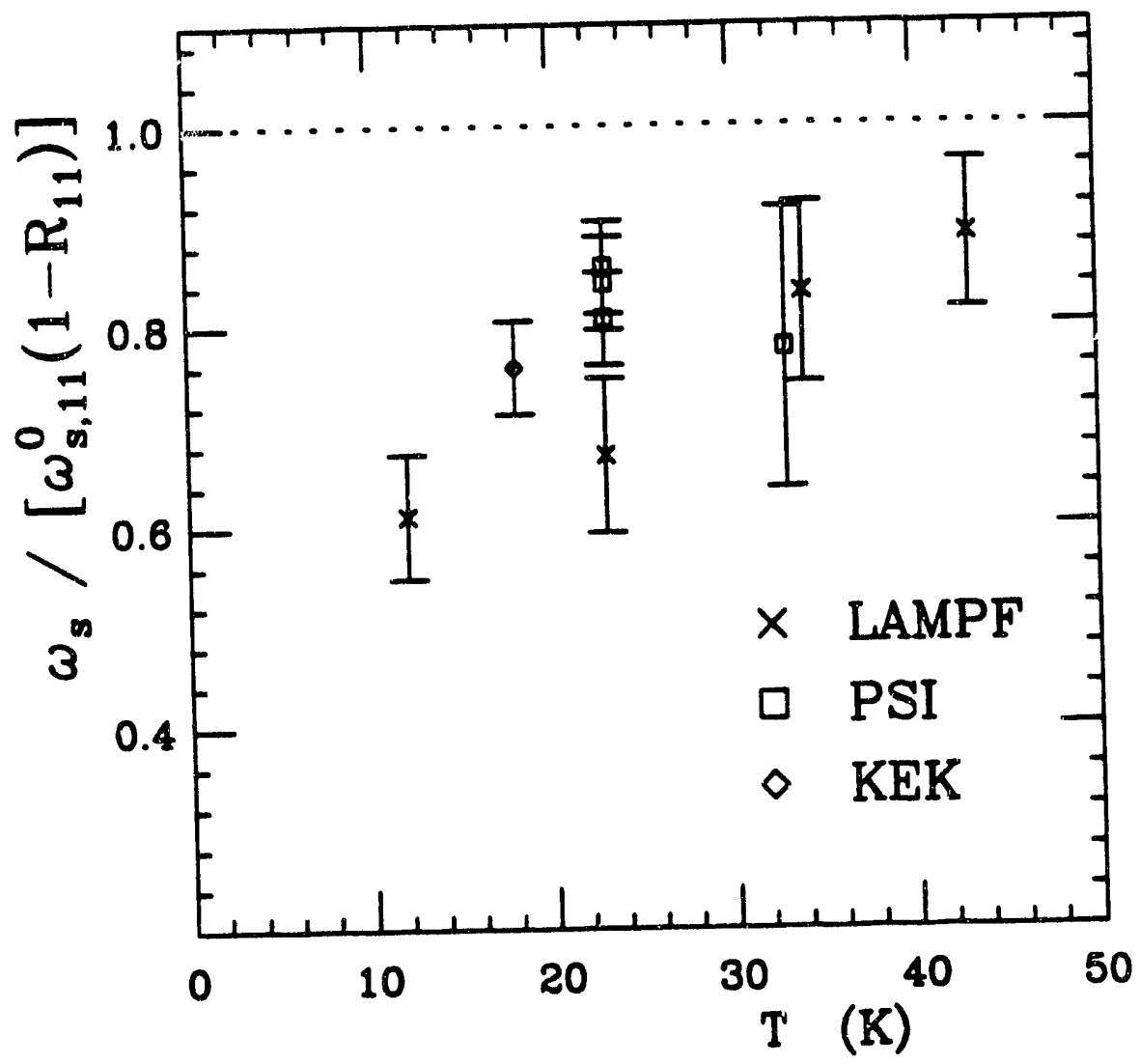

Figure 4: Temperature dependence of the initial sticking fraction. The error bars in the experimental values include uncertainties in the regenerstion factor $R$. 


\section{High density catalyzed fusion}

Muon catalyzed fusion in high density environment was first suggested by [36] and it was subsequently [37] severely criticized. These objections and our current position are as follows [39].

1. Stopping distance of muons in $T>10 \mathrm{keV}$ electron plasma being too long (due to reduced stopping power at sufficiently high temperature). Due to the much enhanced direct fusion rate, the required temperature is 1,000 times lower than considered originally $[36,37]$. Temperature must be chosen wisely such that the stopping distance for muons is sufficiently short, while the regeneration of muons is enhanced $[41,42]$.

2. Rate of formation of muonic hydrogen atoms being too slow and fusion reactions being too slow up to very high temperatures. A detailed study [41] suggests that muonic hydrogen formation is not a bottle neck. We have recently explored [33] direct nuclear fusion reactions, see Section 7

3. IF-confinement time is thought to be much shorter than the required $5 \mu \mathrm{sec}$ - this is a question related to the required densities and temperature. In our opinion temperatures of the order of $10-100 \mathrm{eV}$ are sufficient, as we found new direct fusion mechanisms. Hence the emphasis shifts to the identification of the conditions of density and temperature, target size and its geometry, for which confinement time of $5 \mu \mathrm{sec}$ at $\mathrm{T}=10-100 \mathrm{eV}$ can be achieved. This question will require much further consideration beyond the scope of this project.

The main steps of the MuCF-dt cycle occurring in a D-T mixture are summarized below, adapted to the situation likely to occur in an inertially confined high density plasma target - in order to simplify the situation, we will consider a completely ionized target. Rates given are in most cases normalized to the (atomic) density of liquid hydrogen (LHD) $\rho_{0}=4.2510^{22} \mathrm{~cm}^{-3}$, which is the convenient density scale. We note that when studying to the plasma process one must adjust diverse Auger processes to reflect the modified electron density at the site of the muonic system: normally the center of an electron atom we have:

$$
|\Psi(0)|^{2}=\frac{1}{64 \pi a_{0}^{3}}=50 \rho_{0}
$$

We thus find that at densities well above $50 \mathrm{LHD}$ and $T>30 \mathrm{eV}$ the Auger processes proceed at a rate greater than computed for conventional MuCF atomic processes. In dense plasma we thus have the following MuCF cycle sequence:

1. Muons are stopped within $10^{-10}(\rho / \rho)$ sec in a hydrogen target.

2. Muons are captured into atomic orbits by Auger processes, which usually takes less time than the stopping, with an estimated value $10^{-11}\left(70 \rho_{0} / \rho\right) \sec$ or shorter. Since muons are bound with an energy 207 times greater than electrons, they can form atomic structure at temperatures up to several keV.

3. Muon cascades down by (external) Auger processes induced by two body collisions to the muoatomic L-shell within $10^{-11}\left(\rho_{0} / \rho\right)$ sec. The final transition to the ground state takes less than the radiative rate, which is just about $10^{-11} \mathrm{sec}$, as it is likely that this transition will also be dominated by collision processes, much like the transfer processes. We note that should the muon be captured initially by a deuteron, transfer processes to the heavier isotope compete with the cascade processes in $d \mu$. These transfer processes will be greatly enhanced as compared to conventional MuCF due to three body collisions involving the muoatom and two hydrogen ions. The relative population $d \mu \leftrightarrow t \mu$ is controlled by the Boltzmann factor 
$e^{-\Delta E / T}$, where $\Delta E=48 \mathrm{eV}$ is the energy between the 1s-states in muo-deuterium and muo-tritium. For $\mathrm{T}<48 \mathrm{eV}$ the muon is mostly in the $(t \mu)$ state, at higher $\mathrm{T}$ we have a concentration proportional distribution.

4. The de-excited muoatom collides with another hydrogen ion and undergoes direct fusion reaction at a rate presumably exceeding $10^{9} \mathrm{~s}^{-1}$ at $\rho=1,000 \rho_{0}$. In this respect the IC-MuCF system differs completely from conventional MuCF which proceeds via a chain of molecular processes.

5. If the muon has been captured by (becomes stuck to) the helium produced in fusion, it almost certainly will be regenerated in collisions. The fusion yield is thus mostly limited by reaction rates.

We have studied several direct reaction mechanisms that may occur in IC-MuCF environments [33]. The most immediately obvious is in-flight fusion, in which the Coulomb barrier between the $d$ an $t$ is substantially screened, permitting fusion at low temperatures [36]. At energies below a few $\mathrm{keV}$, tunneling through the barrier is essentially energy independent and the fusion cross section consequently changes like $1 / v$. The resulting fusion rate at $L H D$, which we have computed using an $R$-matrix parameterization of the $d t$ nuclear interaction and which is in substantial agreement with results obtained using optical potentials, is then approximstely [38]:

$$
\lambda_{i \rho}=1 \times 10^{5}\left(\rho / \rho_{0}\right) s^{-1} \quad(0<T<100 \mathrm{eV}) .
$$

This rate scales with density, so at $10^{3}$ LHD we can therefore expect something less than 100 fusions per muon.

A second direct reaction channel which we have studied relies upon the below-threshold amplitudes of the $d t \mu-\alpha n \mu$ continuum, see Section 7. In the dt $\mu$ system, there exists a large and long-ranged leakage of the "fused" an continuum into the $d t$ channel. dt states can fuse by making transitions to this below-threshold quasi-resonant continuum. Typically what we have in mind is the transition of a $d+t \mu$ continuum wave to the below-threshold continuum, following some interaction which permits the transition. The contributing transitions are those with initial and final energies very close to the $d+t \mu$ threshold, with sizes of order $A$, in which case many particles are within the reaction region and many-body reactions become important.

The f:cese we have found to be most favorable at high temperatures is the scattering of $t \mu$ off $d^{+}$, placing the $t \mu$ off mass shell with respect to a second deuteron and enabling the transition to the below-threshold state. Since this is a three-body reaction, the fusion rate scales as $\rho^{2}$. This reaction exhibits a mild but important temperature dependence, favoring low temperatures. The temperature here refers specifically to the temperature of the $d^{+}$ions and the $t \mu$, and in fact most of the fusions come from the low energy part of the thermal distribution. Any deviation from a Maxwellian distribution would have an important impact on the fusion rate. This phenomenon of an increasing fusion rate with decreasing temperature is due to the increasing integrated strength of the below-threshold wavefunction wave in the $d t$ channel as the energy approaches the $d+t \mu$ threshold, in which case it is the low energy part of the thermal spectrum that makes the dominant contribution to the rate.

Another three-body direct fusion reaction may be equally important; this involves the initial scattering of two $d^{+}$ions before one ion fuses with the $t \mu$. In this case, the scattering matrix element is strongly energy dependent, although the dominant contribution comes from the region of the thermal spectrum in which the momentum of the scattering $d^{+}$ions is comparable to the 


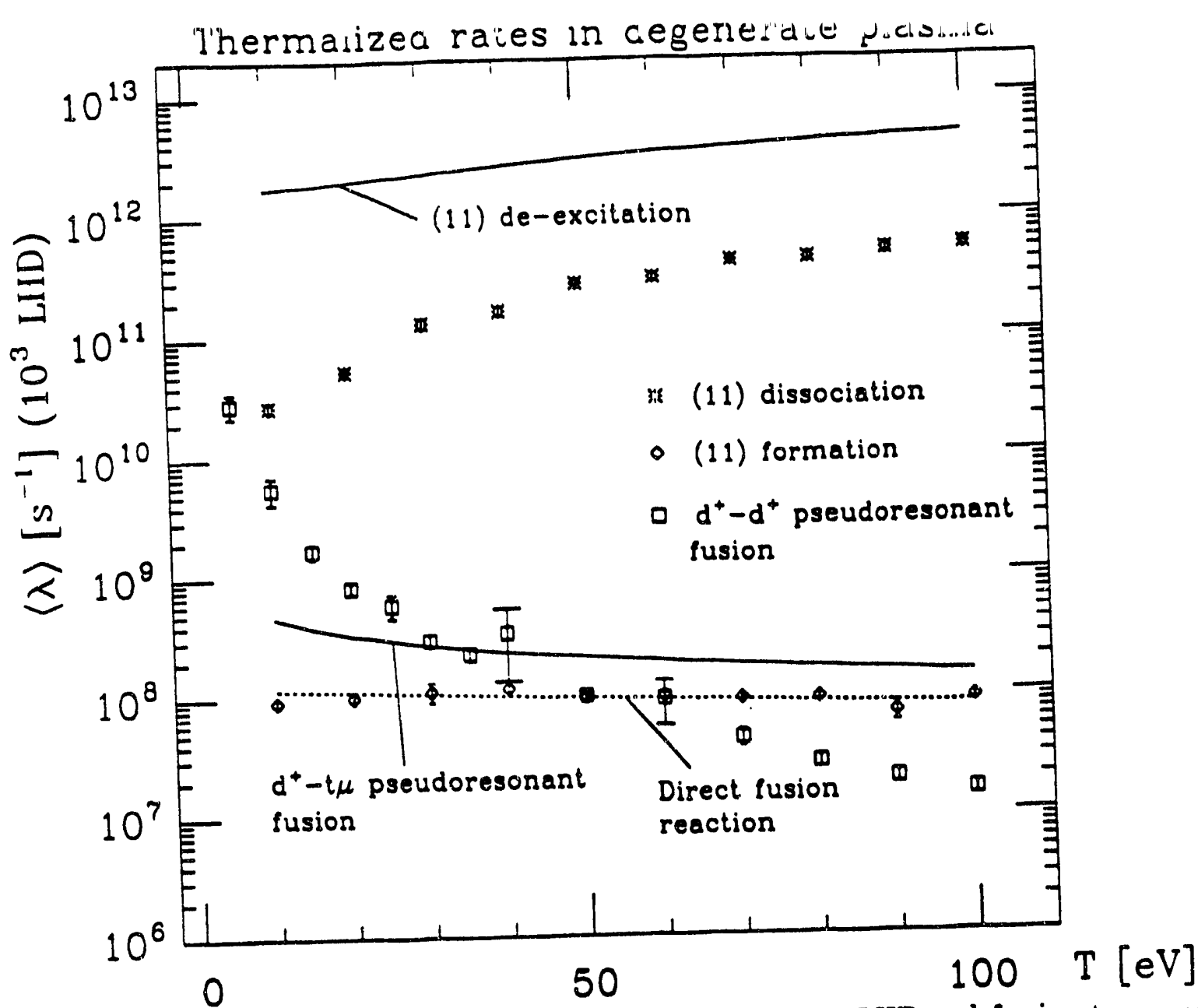

Figure 5: MuCF thermalized plasma fusion rates at density 1,000 LHD and for ion temperature $T_{d}=1-100 \mathrm{eV}$. For details see text. Note that the direct reaction rate scales with density, the "pseudoresonant" rates scale with square of density.

reciprocal of the screening length. In consequence, the fusion rate is more temperature dependent than for the $d^{+}-t \mu$ pre-scattering case. In Figure 5 we show a number of characteristic rates as function of temperature $1<T<100 \mathrm{eV}$ for a dense thermalized degenerate plasma at $\rho=1000 \rho_{0}$. Aside of the rates discussed above we also show the (11) resonance formation and dissociation rate, as well the de-excitation rate. As is apparent, only for temperatures in $10-30 \mathrm{eV}$ are current calculations suggesting the dominance of the novel fusion channels discussed here.

Other direct reactions to be still considered include in particular the formation of deeply bound muonic molecules in three body collisions, as well as the rates of their dissociation, to be compared to fusion rates which for $J=0$ angular momentum states are $10^{11} \mathrm{~s}^{-1}$. It is likely that we did not yet identify the dominant fusion mechanism among the many possible processes in dense matter. On the other hand we have demonstrated here that high density (inertial confinement) MuCF deserves a thorough study as our results suggest that we have identified a viable path of high density and (relatively) low temperature targets in which thousands of MuCF fusions seem possible. We recall that in a degenerate plasma muon sticking is sufficiently reduced by the token of greatly reduced stopping power. The limit to fusion yield in degenerate dense hydrogen plasma is mainly given by the cycling rate and losses due to finite size of the system, both issues presenting considerable future challenge. It is fair to say that in degenerate dense plasma MuCF we are facing a set of problems which are at least as involved as were faced in last 30 years in MuCF at STP. 
We have also investigated the processes involved in muon catalysis of hydrogen isotopes with light nuclei $Z>1$, with the objective of identifying systems in which at least one fusion per muon is possible [43]. A particular practical advantage of such systems is the possible absence of neutrons in the final state, as well as the avoidance of tritium as a reactant. We systematically explored all nuclear systems searching of those having the potential to lead to fast fusion rates despite the high Coulomb barrier. We have considered here in some detail the tunneling through this barrier as well as the internal conversion of the muon. Furthermore, we established in qualitative terms the necessary conditions for muomolecular rates in collisions of muonic atoms of hydrogen isotopes with small concentrations of light elements.

There are two main reasons why MuCF reactions involving $Z>1$ nuclei has previously been dismissed. The first one is that if the muon becomes bound to a $Z>1$ nucleus, it forms a positively charged and therefore inert ion, which will not continue in the chain of fusion reactions. Only if the muon remains attached to a hydrogen isotope, the muonic atom is neutral and can approach closely other nuclei. Hence, only one of the reacting nuclei can bave $Z>1$. The second reason, already noted by Frank [1], is that the barrier penetration calculated in the WKB approximation yields fusion rates more than ten orders of magnitude smaller than those involving hydrogen isotopes only. In our work we bave shown why these arguments are unfounded for certain systems.

Despite some significant advances we do not believe today that muon catalyzed fusion involving nuclei with $Z>1$ is a practicable alternative to $d-t$ fusion; since $Z>1$ nuclei have to participate in the reaction, muon capture (scavenging) cannot be avoided; however, the significance of nonmolecular in flight scavenging can be reduced by working with small partial concentrations $C_{Z}$ of $Z>1$ light nuclei, an approach which is possible if formation of $\left(Z_{\mu H}\right)$ molecules is the dominant reaction due for example to the presence of strong resonances in the material used. In order for $Z>1 \mathrm{MuCF}$ to be of practical interest, a number of requirements must be met:

a) the effective rate for muonic molecule formation must be of order $10^{9} \mathrm{~s}^{-1} / C_{Z}$ to allow for 1000 fusion cycles during the lifetime of the muon;

b) the rate for nuclear fusion must be at least $10^{\circ} \mathrm{s}^{-1}$, but also at least 2000 times the rate of intramolecular transfer reaction of the muon to $\mathrm{Z}$;

c) the probabilities for scavenging of the muon by a $\mathrm{Z}$ nucleus or for capture by a fusion product (sticking) must not exceed $0.1 \%$.

It seems that there is no case of fusion-interest in which these requirements could be fulfilled. However, in order to see one fusion per muon for purely fundamental research interests much more realistic values of the above parameters $\left(10^{6} \mathrm{~s}^{-1}\right)$ arise. Another reason to continue further in this research direction is the need to understand the impact of light element impurities with $Z>1$ in MuCF. MuCF with $Z=2$ ( $\mathrm{He}$ ) has already well studied. Molecular scavenging studies was found to have a rate of $10^{8} \mathrm{~s}^{-1}$.

\section{Fusion catalyzed by ultraheavy charged particles}

An initial reaction to the report of sporadic observation of fusion neutrons by Jones et al [46] in heavy water electrolysis was that cosmic muons are at the origin of these phenomena [47]. The 
primary objection to this suggestion is the well known fact that muons would very quickly become bound to elements other than hydrogen and are removed from the catalytic cycle. Direct experiments [45] have not been able to confirm MuCf as origin of Jones' neutrons. Attention then turned to the possibility of quark catalyzed fusion [48], but it is difficult to understand why, given the relative ease of detecting a fractional charge, it has not been observed at the required level of abundance in matter. Another catalyzed fusion alternative we in particular pursued is the possibility that a yet undiscovered ultra heavy, stable, negatively charged particle $\boldsymbol{X}$ could catalyze the observed fusion reactions [49].

There has been a long standing suggestion by Mamyrin [50] of the possibility that an unknown catalyst is the source of point-like, high abundance of ${ }^{3} \mathrm{He}$ in industrial grade metals, tough other more conservative explanations are also advanced (e.g. tritium migration to metal defects. To present day, the possible existence of such new particle $\boldsymbol{X}$ remains in the realm of a speculative thought. However, should such a particle exist, it would have extraordinary fusion catalyzing properties worth further record. The particular advantage of the large mass $M_{X}$ for the purpose of catalyzed cold fusion is that the inertia of such a particle delays its capture, either because of its immobility, or because of its reduced capture rate by other elements due to the highly increased energy scale. Furthermore, a stable $X$-would bind in the early Universe with the proton, to form a neutral object $\left(X_{p}\right)$ with a binding energy of $25 \mathrm{KeV}$ (assuming for simplicity a pointlike nature for the Coulomb potential) and a size of $30 \mathrm{fm}$. Hence even a substantial cosmic flux of these particles could go undetected till present day, and the detection of their presence, bound with heavier nuclei on Earth requires sensitivity to ultra-heavy "nuclei". Up to now experiments have had little to say about $M_{X}>10^{13} \mathrm{eV}$. Several recent studies $[51,52]$ have pointed out that the existence of $X$ of mass $\mathrm{O}\left(\mathrm{PeV}=10^{16} \mathrm{eV}\right)$ would be most welcome, explaining certain cosmological riddles. These considerations also set upper limits on possible abundance of $X$ in Nature. The flux of such particles is limited by their contribution to the mass of the Universe and is therefore $F<O\left(1 \mathrm{~cm}^{-2} \mathrm{~s}^{-1}\right)$ which for $M_{X} \sim 10^{18} \mathrm{eV}$ would close the Universe. Such a flux would, however, induce observable $X$-ray activity in the outer atmosphere. However, at the level of $10 \%$ of the flux needed to close the Universe, we still would have in these entities as much mass as in the visible Universe, and their flux, would have remained undetectable but can lead to observable electrolysis fusion phenomena.

In our work [53] we have extended considerably considerations of loffe et al [54] regarding the interaction of $x$ in deuterium rich environment, which we believe would be particularly suited to stop the cosmic flux of such ultra heavy particles in the form $\left(X_{p}\right)$. We note that despite our and other studies, the complex chemistry of $X$ is difficult to assess $[49,53,55]$. Despite these uncertainties we can predict that $\boldsymbol{X}$ in a dense deuterium environment will induce catalyzed fusions, and this constitutes a sensitive method of detecting a primordial fux of superheavy charged particles. The particular systematic advantage of a search for $\boldsymbol{X}$ in deuterium rich environments derives from the fact that neither the precise knowledge of all interactions between $X$ and hydrogen, nor the mass of $X$ are crucial parameters for a large range of values. In our view $[49,56,53]$, the fate of $(X p)$ impinging on materials with even a tiny deuterium abundance is as follows:

1. $\left(X_{p}\right)$ becomes $(X d)$ following a hydrogen exchange reaction on deuterium. Since in this process $25 \mathrm{keV}$ energy is released, the relevant cross section is very large and we believe that this process is by far the dominant channel for an exchange reaction of $X_{p}$ with nuclei in matter.

2. Once $(X d)$ is formed, it has an appreciable probability of inducing diverse $(d, p)$ or $d-d$ nuclear fusion reactions. Energy released in such interactions leads to the removal of the hydrogen remnant from the $X^{-}$atomic orbit.

3. Subsequently, a negatively charged $X^{-}$will pass through the matter, leaving behind a track 
of ionization energy. The substantial relative kinetic energy of $M_{X}$, which may be as high as $500 \mathrm{MeV}$ and which arises from the motion of the Earth through gas of these neutral objects presumed to be nearly stationary in the Universe, by virtue of their large mass.

4. The reason that a flux of $(X p)$ could have gone undiscovered may be in part due to a probably small chance of $X$ reaching the Earth's surface. The chance of $X$ reaching the Earth's surface in its active form $(X p)$ or $(X d)$ is potentially a function of numerous environmental factors, due to the complex pattern of interactions $X^{-}$can enter with different materials in even trace abundances.

In conclusion, while we have pointed [49] to the possible relation between the Jones' report [46] of neutron activity in electrolysis with natural flux of yet undiscovered $X$ particles, the question if this is the source of the observed anomaly is purely experimental, and so far there seems to be no positive evidence to confirm this proposal. On the other hand, from this work arises the insight that fusion activity is a sensitive probe for the flux of ultra heavy, negatively charged particles [53], and that existence of such entities would very probably make catalyzed fusion viable.

\section{Muon production}

Any MuCF system we deaign will effectively be an energy multiplier as without an input (in form of the catalyst) it can not function. Consequently, it is clear that there is a lot of practical leverage in reducing the energy cost of muon production. We also note that muons need to be formed in large quantities since even if each muon were to catalyze thousands of fusions, the thermal power would be $1.510^{-3} \mathrm{~W}$ per muon during its lifetime (assuming 1000 fusions per muon). Said differently, in order to generate the continuous thermal power of only $1.5 \mathrm{MW}$, we need a steady presence of $10^{\circ}$ muons, that is a usable muon flux of $10^{\circ} / 2.2 \times 10^{-8} / \mathrm{s}=70 \mathrm{nA}$ muons. The muon production issue can be summarized as follows: how does one produce $\mu A$ fiuxes of usable muons at an energy cost which is not greater than few GeV beam power per muon? The practical path to produce high intensity cheep muon beams as required in MuCF is believed to proceed via hadronic interactions, that is the production of negative pion beams in collisions of (neutron-rich light) nuclei which in vacuum always decay into the needed negatively charged muons. In addition to the composition of the beam and target, the $\pi^{-}$production cost depends also on the geometry of the target, projectile beam energy, and the external fields used to channel pions out of the target volume.

In our at this stage conceptual, but quantitative work we have invoked off-the-shelf technologies only, when considering the energy cost of muon production. It should be remembered that a usable muon is a muon which has been stopped within the MuCF fusion vessel. There are two principally different concepts which we considered:

1. the active target scheme: a fixed target is external to the accelerator, and production of muons for fusion occurs in immediate vicinity of the fusion vessel, the muon (pion) production target is practically the fusion device. Such a concept is in principle similar to the spallation breeder concept and similar ideas have been put forward to merry muon catalyzed fusion with fast breeder - - we have rejected this line for pragmatic reasons and were lead to consider:

2. the storage ring with internal target: the beam of particles which contains the energy to be used for muon production is stored in a ring which contains a thin primary target - secondary beams emanating from the interaction of the stored beam with the internal target are either directly or indirectly employed in shaping a suitable muon beam - considerable advantage is 


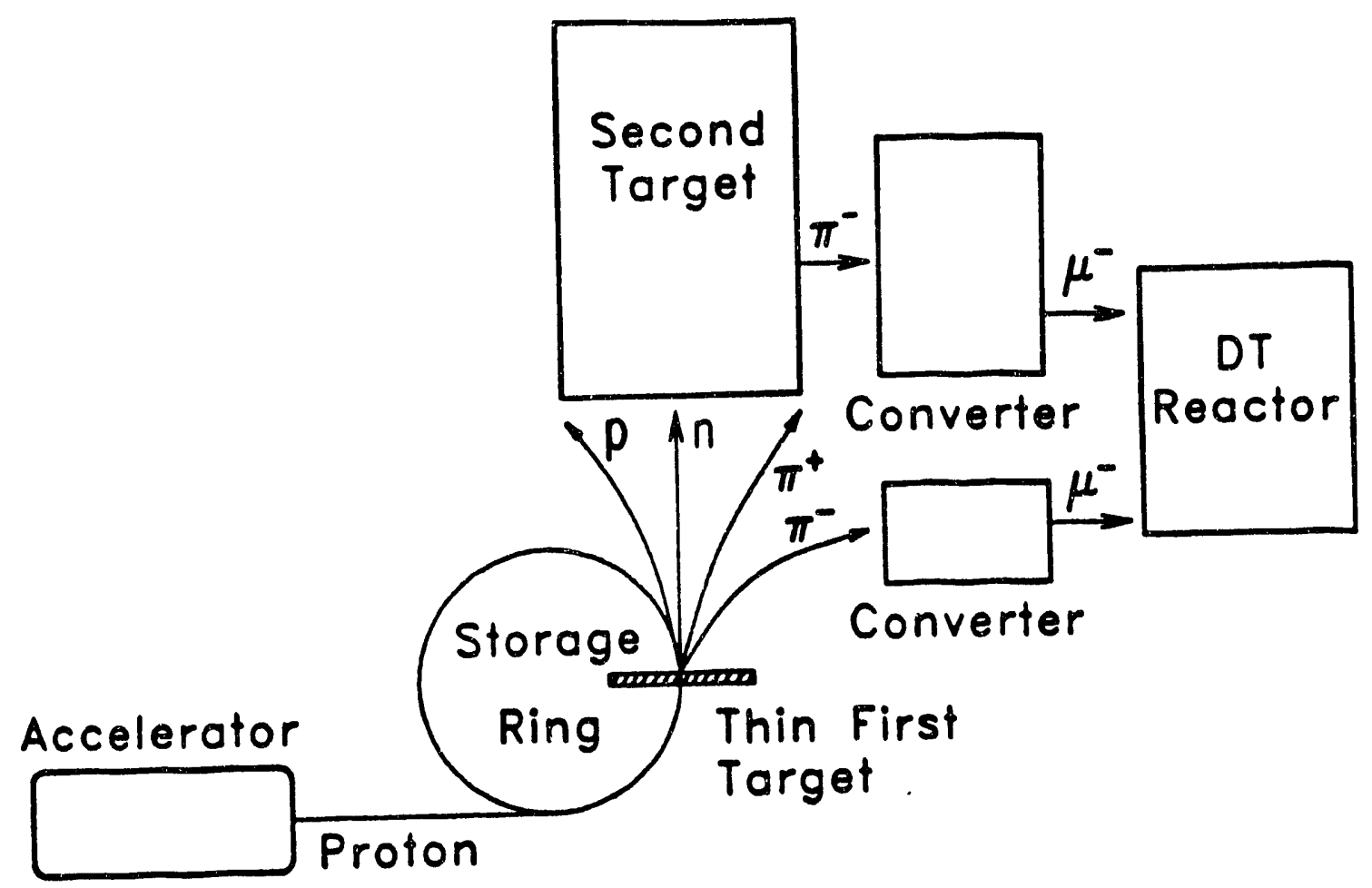

Figure 6: Scheme of a MuCF reactor.

derived in such an approach from both the presence of the negative pions in the secondary beams as well as from the diversity of the secondaries originating in a well defined interaction vertex.

In detail, the scheme [57] which we believe is most practical consists of a storage ring, a thin internal beryllium target and several thin external targets, see Figure 6. The stored proton beam $(5 \mathrm{GeV})$, chosen also to minimize beam losses produces as de of the primary $\pi^{-}$also other usable shower particles. Shower particles are directed to external target to produce more negative pions. To evaluate the effectiveness and cost of pion production we carried out simulations with a hadronic cascade Monte-Carlo code. We have estimated the negative pion production cost for a number of metallic internal targets, such as Be, $\mathrm{Pb}, \mathrm{AB}$. Cu and $\mathrm{Al}$. We have studied the secondary shower particle distribution as function of energy, momentum and aximuthal angle for these metals as the internal target materials. We found that the energy cost, about $11 \mathrm{GeV}$ beam energy per first interaction negative pion are comparable to other proposed schemes. However, we found that it is possible to greatly reduce this cost by use of the secondary beams emerging from the thin internal target. Including $\pi^{-}$production by secondary shower particles from the internal target having an azimuthal angle greater than $36^{\circ}$, we estimate an energy cost of about $2.7 \mathrm{GeV}$ per negative pion for an initial beam momentum of $5 \mathrm{GeV} / \mathrm{c}$, ' 1 sing beryllium as internal and external target material.

Even though there is no convincing evidence as yet that MuCF will ever become a viable energy alternative, several workers, in recognition of the observation that the fusion yield and cost of energy production are strongly system dependent, began the conceptual MuCF-fusion reactor design. Fundamentally all such work centers around Petrov-MuCF reactor system [58]: a hybrid system consisting of a MuCF fusion reactor with a plutonium breeder reactor. This system includes an accelerator (for $d$ or better $t$ ), a pion-producing target, a convertor and blankets. Our work, however, suggests an alternate approach. Rather than to multiply MuCF energy yield by a breeder 
reactor, we think that it is possible to increase the fusion yield in particular in extreme conditions prevailing in degenerate hydrogen plasma.

\section{Studies of nuclear cold fusion}

Even before the report by Jones [46] of the possible observation of nuclear fusion during electrolysis of heavy water there has been some interest in the possibility that fusion could be catalyzed by ordinary electrons in non-equilibrium conditions [59]. Spontaneous fusion of $D_{2}$ molecules at an observable rate was dismissed in a study by Van Siclen and Jones [60], who estimated a fusion rate in the $D_{2}$ molecule of less than $10^{-70} \mathrm{~s}^{-1}$. Following on the experimental report, we have considered in some detail conditions which my have lead to the result. The effects considered [61] were space confinement, electron screening, and/or some collective mechanisms which could effectively increase the mass of the electron or lead to en enhancement of the deuteron relative energy. It was further recognized that diverse solid state effects greatly influence the interaction potential and hence the fusion rate [62]: the high electron densities available in metal hydrides lead to a substantial decrease of the long range Coulomb potential, particularly for long distances. Hence even though the mean separation between the hydrogen nuclei in a metallic lattice is larger than it is in the usual molecule, the fusion rate can be considerabiy greater, since metallic electrons form a electron plasma and plasma density fluctuations pro ride a natural screening of the long range Coulomb potential:

$$
V=-Z_{1} Z_{2} e^{2} \frac{e^{-r / r_{0}}}{r} \text {. }
$$

If $r$, derives from the density of states of the conduction electron gas, we find that $r$, can be as small as $0.15 \AA$ for palladium and $0.25 \AA$ for titanium, taking the density of states from the heat capacity of electrons. In titanium, the equilibrium separation between two neighboring hydrogen sites is significantly smaller than in $\mathrm{Pd}$, making it potentially the more interesting material for fusion studies in condensed matter.

The two body fusion rate at low energies is mainly controlled by the tunneling through the repulsive Coulomb like barrier, and this process is controlled by the parameter $\eta$ which in WKB approximation is given by:

$$
\eta=\frac{1}{x} \int_{r_{\text {min }}}^{r_{\text {max }}} \sqrt{2 \mu(V(r)-E)} d r .
$$

The range of the iteration is in the classically forbidden region of motion, where the root is positive definite. $\mu$ is the reduced mass pertinent to the relative motion of the reacting bodies, $V$ is their (repulsive) potential and $E$ is the relative energy. For the Coulomb potential, $\eta$ reduces to the usual Sommerfeld parameter, i.e. $\eta_{c}=\alpha /(v / c)$ where $\alpha=1 / 137$ is the fine-structure constant. The critical role of $\eta$ is best illustrated by recalling the Gamov form for the nuclear reaction cross sections:

$$
\sigma(E)=\frac{S(E)}{E} e^{-2 \eta\left(E ; r_{0}\right)} .
$$

Here, $S$ is the so called astrophysical factor, which in absence of threshold resonances is a slowly varying function of energy. For energies of a few $\mathrm{keV}$, the $d$-d-fusion neutron $S$-function is measured to be about $53 \mathrm{keV}$ barn, and in free space $\eta=\beta / \sqrt{E}$, where for $E$ measured in $\mathrm{keV}, \beta=44.4021$ $(\mathrm{keV})^{\frac{1}{2}}$. It is easy to see that to reach Jones' fusion rates energy scale of $a$ few $100 \mathrm{eV}$ is required.

We note that the conventional expression Eq.(26) fails in principle for low energies: while all inelastic cross sections must diverge as inverse of the relative velocity (screened Coulomb potential), the above expression for a screened Coulomb potential diverges as $1 / v^{2}$. We have therefore 


\section{dd fusion cross section}

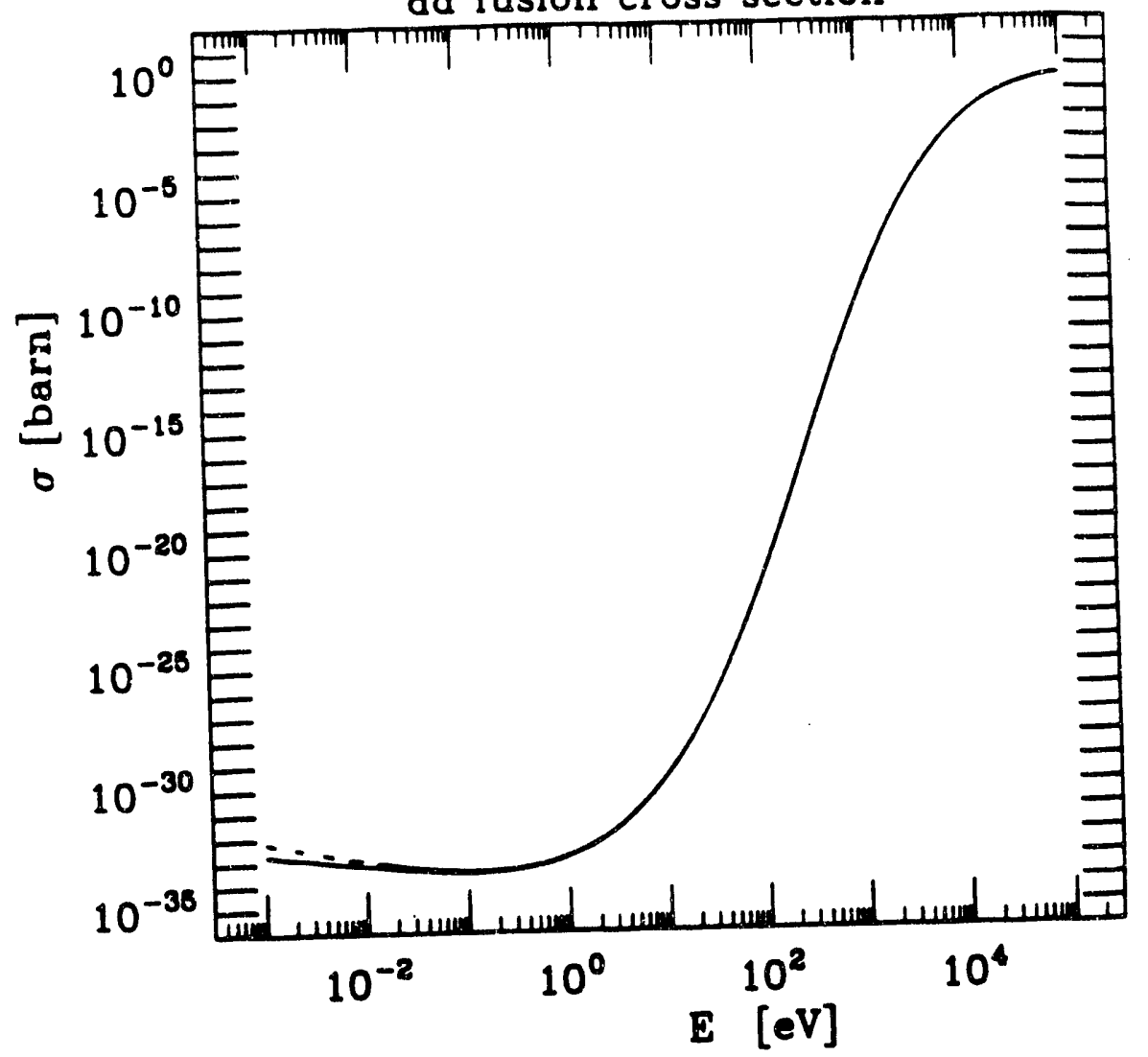

Figure 7: $d(d, n){ }^{3} \mathrm{He}$ cross section for screening radius $r_{0}=0.05$ Ain a reaction model $[63]$.

devoted much effort to developed a correct and sophisticated nuclear reaction description [63] of low energy $d-d$ fusion process. We indeed found that while for ultra low energies the discrepancy we noted is corrected, the Gamov formula Eq.(26) applies when the rates of reactions reach the here interesting values. The situation is illustrated in Figure 7 where the $d(d, n)$ crose section computed for screened potential in reaction model is shown for the over screening used, $r_{1}=0.05 \AA$. The dashed line is the result of the astrophysical formula, the full line is the reaction model. One should be cautioned not to take the magnitudes of these extremely small fusion cross section too seriously, since there is almost certainly some yet unaccounted for higher order or collective process at such a small level. Our final conclusion is that it is not possible to explain Jones' neutron production in terms of two body reactions [64].

Consequently, only non-equilibrium, collective many body phenomena could have lead to the fusion rate reported. Jones's cold fusion must be a quantum process, far away from the classical limit. Especially in a near degenerate condensed matter environment a theory must allow for many body non - equilibrium quantum phenomena. Yet estimates of fusion rates as briefly reported above are based on classical two body free space reactions. Note that despite the fact that individual deuterons are classical objects, a part of their amplitude is in the quantum domain (otherwise they could not fuse at all) and the question arises about the coherence of the small quantum tails of the $\mathrm{N}$-body wave function. This issue may be greatly amplified where non-equilibrium phenomena are involved. Only a non-equilibrium study of dynamic quantum - two - plasme model can provide the ultimate theoretical limit on a conventional cold fusion rate in condensed matter. Such a project presents formidable theoretical challenges. 
Another question which arose in the context of Jonses' experiment was the issue which is the more sensitive measure of rare fusion activity: the laboratory experiment, or the astrophysical limits arising in particular in studies of properties of ultramassive planets, such as Jupiter. At least in part, the impetus to study fusion in condensed matter came from the association of the high preanure in this planet with fusion rates $[60)$. These results were reconsidered soon after cold nuclear fusion phenomens carne to public attention [65]. We have carefully evaluated [66] within a realistic Jupiter model $[68,67,69]$ the fusion yield, including the ascumulated fusion yield over the history of the planet. One of the reasons we are interested in temporal evolution is that at these small temperatures nuclear fusion reactions are recognized by the accumulation of ${ }^{3} \mathrm{He}$ and hence a changed isotope ratio of ${ }^{9} \mathrm{He}$ to ${ }^{4} \mathrm{He}$.

The exiating theories of the Jupiter are constrained by the few observational properties of this planet. The moat inportact constrains are: Mass: $M_{J}=0.00095 M_{\odot}$; Radius: $R_{J}=7.149 \times 10^{9}$ $\mathrm{cm}$; Age: $t_{J}=4.5 \times 10^{9}$ years; Luminosity: $L_{J}=7.04 \times 10^{24} \mathrm{erg} / \mathrm{sec}$, which is about twice as much energy a it absorbs. We note that present densities of hydrogen and temperatures at Jupiter are bout 50 times higher than those in metallic hydrides explored in experiments. While the temperature is also $\mathbf{5 0}$ times larger than the room temperature, the mean hydrogen collision energy on Jupiter it is atill significantly below the lowest energy accessible to laboratory experiments today. In the atandard model the intrinsic thermal radiation from Jupiter is explained in terms of the gravitational contraction and cooling of a solar type composition object of Jovian mass. However, these modela leave ample opportunity for much of the heat to be generated by other sources.

We used the resulta of the spherically symmetric model of Jupiter based on Ref. [69]. The planet is asoumed to be fluid throughout, completely convective except for a thin radiative zone in the atmosphere, completely adiabatic, and fully mixed. In the convective core (about $80 \%$ by radius) electrons are degenerate. The surface consists mainly of molecular bydrogen, and as it is a small fraction of Jupiter's mase, it does not matter in our considerations. The standard model results imply that the central density, $4 \mathrm{~g} / \mathrm{cm}^{3}$, corresponds to about $2 \times 10^{24}$ hydrogen atoms per cubic centimeter and the central temperature is about $1.4 \mathrm{eV}$ - and both these quantities change very slowly incide Jupiter. Under these conditions our conventional wisdom calculation suggest that the fusion yield is dominated by $p-d$ reactions and is 17 orders of magnitude ahort in explaining the luminosity of the Jupiter.

We than examined nuclear resction rates assuming that the interior of Jupiter is twice hotter than it in actually believed to be, i.e. the central temperature is about $2.8 \mathrm{eV}$. This assumption does not violate the basic physics of Jupiter, in particular the equation of state of degenerate electron gas. The Fermi energy of electrons is estimsted to be about $E_{f} \sim 50 \mathrm{eV}$, and is still larger than the assumed central temperature. In comparison with the standard Jupiter model the d-d fusion rate is increased by more than four orders of magnitude, but p-d fusion is still largest by seven orders of magnitude and we are still about 13 orders of magnitude away from a significant contribution to Jupiter's heat. On the other hand we found that should the interior pressure be twice the currently believed value, the fusion energy reaches the thermal yield of Jupiter. This occurs since such a change in pressure induces a drastic change in temperature profile of the planet. it is not believed that such a grose change of standard Jupiter model is permissible.

At this poizt we note that we used theoretical reaction cross sections which would never lead to obeervable nuclear phenomena in metallic hydrides. So imagine that for some yet unexplored reason, all considered nuclear processes at ultra low energies would have been enhanced by a factor 
- of $10^{17}$, the excess heat of Jupiter could than be easily attributed to "cold" p-d fusion. We also find that such an enhancement of the fusion rate would lead to an enhanced ${ }^{3} \mathrm{He}$ to ${ }^{4} \mathrm{He}$ ratio in Jupiter. Moreover we can question what happens if we assume that $d-d$ fusion in Jupiter occurs with a rate inferred by Jones i.e., which means that $\langle\sigma \cdot v\rangle_{d d}$ is enhanced by a factor of $10^{16}$. Then the rate of energy production in Jupiter due to the $d-d$ fusion is still nine orders of magnitude smaller than the observed luminosity of Jupiter. Thus Jones' experiment is by so much a more sensitive probe of rates of $d-d$ fusion reactions.

We have thus determined that the Jones's experiment [46] is not in any contradiction with available Jovian data and that indeed the experiment is a more sensitive probe of fusion activity, considering both time evolution and the present day Jupiter conditions. More generally, we found no physical environment with which Jones' result [46] for sporadic neutron production in heavy water electrolysis would be inconsistent, even though we have not been able to find an explanation for the phenomenon: we are left with questions relating to possible catalytic activity of a yet undiscovered particle $\boldsymbol{X}$ and possibly collective, off-equilibrium quantum behavior in metallic hydrides under electrolysis.

\section{Principal publications}

Listed here are publications co-authored by the principal investigator in the Geld of Energy Re. lated Applications of Elementery Particle Physics which were prepared under the auspices of the bere reported program in the period February 1988 - Msy 1991. This list contains aside of refereed papers, significant unrefereed work such as major conference and laboratory reports, books and chapters in books, which when appropriate are placed in direct relation to related refereed work.

\section{GENERAL REVIEWS PUTTING THE WORK INTO GREATER PERSPECTIVE}

- Proceedings of the 1988 Meeting on "Muon Catalyzed Fusion", S.E. Jones, H. J. Monkhorst and J. Rafelski, eds., AIP Proceedings series No. 181, New York, 1989.

- "The Challenges of Muon Catalyzed Fusion," in Muon Catalyzed Fusion 1988, AIP Proceedings Series No. 181, p 451, New York, 1989.

- (a) "Review of the Current Status of Cold Fusion" (with D. Harley and M. Gajda), in The Nuclear Equation of State: Discovery of Nuclear Shock Waves and the EOS, W. Greiner and H. Stöcker eda. (Plenum, New York, 1990), p 541.

(b) "Review of the Current Theoretical Status of Cold Fusion" (with D. Harley and M. Gajda), in Emerging Nuclear Energy Systems 1989, U. von Möllendorf and B. Goel, eds. (World Scientific, Singapore, 1989) p 308.

- "Cold Fusion: Muon Catalyzed Fusion" (with H.E. Rafelski, D. Harley, G. R. Shin), J. Phys. B: At. Mol. Opt. Phys. 24, (1991) 1469.

- "Muon Catalyzed Fusion" (with H.E. Rafelski), in Advances in Atomic, Molecular and Optical Phyoics, Vol 29, (1991), p 177, D.R. Bates and B. Bederson, eds..

- "Muons in Fusion" (with H. E. Rafelski), Particle World 2, (1991) 21. 


\section{MUON PRODUCTION}

- "Active Target Production of Muons for Muon Catalyzed Fusion" (with M. Jändel and M. Danos), Phys. Rev. C37, (1988) 403.

- (a) "Deep Antiproton Annihilation on Nuclei," in addendum to Physics at LEAR with Low Energy Antiprotons, C. Amsler, et al, eds. (Harwood Academic Publishers, Chur and London, 1988).

(b) "Deep Antiproton Annihilation on Nuclei", in Antiproton-Nucleon and Antiproton-Nucleus Interactions, F. Bradamante, J.-M. Richard and R.Klapisch, eds. (Plenum Press, New York, 1990), p 231.

- "Quark Gluon Plasms in $4 \mathrm{GeV} / \mathrm{c}$ Antiproton Annibilations on Nuclei," Phys. Lett. B207, (1988) 371.

- "Quark Gluon Plasma in Antiproton Annihilation on Nuclei," in Proceedings of the Prd Conference on the Intersection between Particle and Nuclear Physics, Rockport 1988, G. Bunce, ed., AIP Proceedings Series No. 176, p 393, New York, 1988.

- "Pion and Negative Muon Production for Muon Catalyzed Fusion," (with M. Jändel and M. Danos), Muon Catalyzed Fusion 3, (1988) 557.

- "Double Target Option for Pion Production for Muon Catalyzed Fusion" (with G.R. Shin), in Emerging Nuclear Energy Systems 1989, U. von Möllendorf and B. Goel, eds. (World Scientific, Singapore, 1989) p 271.

- "Pion Production for MuCF" (with G. R. Shin), Nucl. Instruments and Methods A287, (1980) 565.

\section{DIRECT CATALYZED FUSION REACTIONS}

- " $(t \mu)+d$ in Flight Fusion" (with D. Harley and B. Müller), in MuCF 1989, Rutherford Appleton Laboratory 90.028, 1990,p 56; J.D. Davies, ed..

- "Time Independent Description of the $t(d, n) \alpha$ Fusion Reaction in the Presence of the Muon" (with D. Harley and B. Müller), Z. Physik A336, (1990) 303.

- "Nonresonant Fusion Reactions in the $t \mu+d$ System" (with D. Harley), Muon Catalyzed Fusion 5/6, (1990/1991) 249.

\section{FATE OF MUONS AFTER FUSION}

- "Muon Sticking in Muon-catalyzed d-t Fusion" (with H. E. Rafelski, B. Müller, D. Trautmann, R. D. Viollier and M. Danos, Muon Catalysed Fusion 1, 315 (1987).

- "Nuclear Resonance Effects on the Muon Sticking Probability in Muon Catalyzed D-T Fusion" (with M. Danos and B. Müller) Muon Catalyzed Fusion 3, (1988) 443.

- "Muon Reactivation in Muon Catalyzed D-T Fusion" (with H. E. Rafelski, B. Müller, D. Trautmann, and R. D. Viollier) in Progress in Particle and Nuclear Physics 22, (1989) 279. 
- (a) "Muon Spectrum and Convoy Effects after Muon Catalyzed Fusion" (with B. Müller and H.E. Rafelski), Phys. Rev. A.40, (1989) 2839.

(b) "Muon Spectrum and Convoy Effects After Muon catalyzed Fusion," (with B. Müller and H. E. Rafelski), in MuCF 1989, Rutherford Appleton Laboratory 90-022, 1990, p 81; J.D. Davies, ed..

- "Radiative Muon Capture in Light Atoms" (with G. Soff), Z. Physik D14, (1989) 187.

- "Muons After d-t Fusion" (with G.R. Shin), J. Phys. G16, (1990) L187.

- "Muons After d-t Fusion (comment)" (with G.R. Shin), Phys. Rev. A43, (1991) 601.

- "Transport Theory Description of the Muon after $d-t$ Fusion" (with G.R. Shin and H.E. Rafelski), Muon Catalyzed Fusion 5/6, (1990/1991) 315.

\section{NOVEL CATALYZED FUSION OPTIONS}

- "Possible Influence of Vacuum Polarization on $Q_{1}$, in Muon Catalyzed D-T Fusion" in Muon Catalyzed Fusion 1988 (with B. Müller M. Jändel, and S. E. Jones), AIP Proceeding Series No. 181, p 105, New York, 1989.

- (a) "Muon Catalyzed Fusion of Nuclei with $Z>1^{\text {" }}$ (with D. Harley and B. Müller), J. Phys. G: Nucl. Part. Phys. 16, (1989) 281.

(b) "MuCF with $Z>1$, " in Muon Catalyzed Fusion 1988 (with D.Harley and B. Müller), AIP Proceedings Series No. 181, p 239, New York, 1989.

- "Muon Catalyzed Fusion at High Density" (with D. Harley), Particle Accelerators 37/38, (1991) 409.

\section{COLD FUSION AND ITS CONSISTENCY WITH NUCLEAR PHYSICS}

- (a) "Observation of Cold Nuclear Fusion in Condensed Matter," (with S.E. Jones, E.P. Palmer, J.B. Czirr, D.L. Decker, G.L. Jensen, J.M. Thorne, and S.F. Taylor), Nature 338, (1989) 737.

(b) "Anomalous Nuclear Reactions in Condensed Matter: Recent Results and Open Questions" (with S.E. Jones, E.P. Palmer, J.B. Czirr, D.L. Decker, G.L. Jensen, J.M. Thorne, S.F. Taylor), Fusion Technology 19, (1990) 199.

- "Limits on Cold Fusion in Condensed Matter: a Parametric Study" (with M. Gajda and D. Harley), University of Arizona Report AZPH-TH/89-19-2 (broadly circulated, unpublished).

- "How Cold Fusion Can be Catalyzed" (with M. Sawicki, M. Gajda and D. Harley), Fusion Technology 18, (1990) 136.

- "Attenuation of the Flux of Neutrochamps in the Earth's Atmosphere - A parametric Study" (with M. Sawicki), J. Phys. G18, (1990) L197.

- "Reactions of charged massive particles in a deuterium environment" (with M. Sawicki, M. Gajda and D. Harley), Phys. Rev. A44, (1991) 4345. 
- (a) "Jovian Limits on Conventional Cold Fusion" (with M. Gajda), J. Phys. G: Nucl. Part. Phys. 17, (1991) 653.

(b) "Nuclear Fusion in Jupiter" (with M. Gajda), in AIP proceedings series, Vol. 228 (1991) p 591, S. E. Jones et al, eds.

- "Coupled Channel Model for Ultra-Low Energy Deuteron-Deuteron Fusion" (with W. Żakowicz), in AIP proceedings series, Vol. 228 (1991) p 911, S. E. Jones et al, eds.

\section{References}

(1) Frank F C, Nature 160, (1947) 525.

[2] Alvares L W, Bradner H, Crawford Jr. F S, Crawford J A, Falk- Vairant P, Good M L, Gow J D, Rosenfeld A H, Solmits F, Stevenson M L, Ticho H K and Tripp R D, Phys. Rev. 105, (1957) 1127.

[3] Rafelski J and Rafelski H E: "Muon Catalysed Fusion" in Advances in Atomic, Molecular and Optical Physics, Vol 29, (1991), p 177, D.R. Bates and B. Bederson, eds..

(4) Jones S E, Anderson A N, Caffrey A J, Van Siclen C D W, Watts K D, Bradbury J N, Cohen J S, Gram P A M, Leon M, Maltrud H R, and Paciotti M A, Phys. Rev. Lett. 86, (1986) 588.

[5] Hartmann F J, Baumann P, Daniel H, Grunewald S, Lipowsky R, Moser E, Neumann W, Plendl H S, Schmidt G, Schott W, von Egidy T, Ackerbauer P, Breunlich W H, Cargnelli M, Fuchs M, Jeitler M, Kammel P, Marton J, Nägele N, Werner J, Zmeskal J, Bosay H, Crowe K, Sherman R H, Lou K, Petitjean C, Muon Catalysed Fusion 5/6, (1990/91) 277.

(6) Breunlich W H, Cargnelli $M$, Kammel $P$, Marton N, Nägele N, Pawlek $P$, Scrinsi $A$, Werner J and Zmeskal J, Phys. Rev. Lett. 88, (1987) 329.

(7) Nagamine K: Muon Catalysed Fusion 1, (1987) 137.

(8) Bosay H, Daniel H, Hartmann F J, Neumann W, Plendl H S, Schmidt G, von Egidy T, Breunlich W H, Cargnelli M, Kammel P, Marton J, Nägele N, Scrinsi A, Werner J, Zmeskal J and Petitjean C, Phys. Rev. Latt. 59, (1987), 2864.

[9] Balin D V, Baturin V N, Chestnov Yu A, Dyin A I, Maev E M, Petrov G E, Petrov L B, Semenchuk G G, Smirenin Yu V, Vorobyov A A, Vorobyov An A and Voropaev N I, Muon Catalysed Fusion 5/6, $(1990 / 91) 481$.

[10] Rafelski H E, Harley D, Shin G R, and Rafebki J, J. Phys. B: At. Mol. Opt. Phys. 24, (1991) 1469.

(11) Jackson J D, Phys. Rev. 106, (1957) 330.

[12] Rafelski hI E, Müller B, Rafelski J, Trautmann D, Viollier R D and Danos M, Muon Catalysed Fusion 1, (1987) 315.

(13) Shin G R and Rafelaki J, J. Phys. G16, (1990) L187.

[14] Shin G R and Rafeleki J, Phys. Rev. A43, (1991) 601.

[15] Shin G R, Rafelaki H E, and Rafelski J, Muon Catalysed Fusion 5/6, (1990/1991) 315.

[16] Ceperley D and Alder B J, Phys. Rev. As1, (1985) 1999.

[17] Hu C-Y, Phys. Rev. As4, (1986) 2536.

(18) Haywood S E, Monkhorat H J and Szalewics K, Phys. Rev. As7, (1988) 3393.

[19] Haywood S E, Monkhorat H J and Szalewics K, Phys. Rew. As9, (1989) 1634. 
- (20) Jesiorski B, Moskynski R, Ssalewics K, Scrinzi A, Zhao X, Kolos W and Velenik (1990), University of Deleware, Newark. Unpublished.

[21] Kamimura M : "Effect of Nuclear Interaction on Muon Sticking to Helium in Muon Catalyzed d-t Fusion" in Muon Catalyzed Pusion 1988, AIP Proceedings Series No. 181, p 330, New York, 1989.

[22] Rafelaki H E and Müller B, Comp. J. Phys. Comm. 59, (1990) 521.

[23] Rafelaki H E, Müller B, Rafelski J, Trautmann D, Viollier R D),Progress in Particle and Nuclear Physics, 22 (1989) 279.

[24] "Muon Regeneration in Degenerate Hydrogen Plasma", Harley D., to be published.

[25] Kularud R M : "A Proposed Method for Reducing the Sticking Constant in M.C.F." in Muon Catalyzed Fusion 1988, AIP Proceedinge Series No. 181, p 367, New York, 1989.

(26) Stodden C D, Monkhorst H J, Ssalewics K, Winter T G, Phys. Rev. A11, (1990) 1281.

[27] Nagamine K, Muon Catalysed Fusion 5/6, (1990/1991) 289.

(28) Cohen J S, Muon Catalysed Fusion 8, (1988) 421.

(29) Markushin V E, Muon Catalysed Fusion 2, 395 (1988).

[30] Takahashi H, Muon Catalysed Fusion 2, (1988) 453.

[31] Anderson A N: "Investigation of $q_{10}$ ", in Muon Catalysed Fusion AIP Procedinge Series No. 181, p 57, New York, 1989.

[32] Jones S E, private communication.

[33] Harley D and Rafeleki J, Muon Catalysed Fusion 5/6, (1990/1991) 249.

[34] (a) Müller B, Rafelski H E, and Rafelski J, Phys. Rev. A10, (1989) 2839.

(b) Müller B, Rafelaki H E, and Rafeloki J: “Muon Spectrum and Convoy Effects After Muon catalysed Fusion," in MuCF 1989, Rutherford Appleton Laboratory 90-082, 1990, p 81; J.D. Davies, ed..

[35] Soff G and Rafelski J, Z. Physit D14 (1989) 187.

[36] Tan W P S, Nature 268, (1976) 656.

[37) Hincks E P, Sundaresan M K, Watson P J S, Nature 269, (1977) 584.

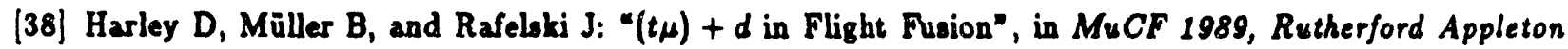
Laboratory 90.082, 1990, p 56; J.D. Davies, ed..

[39] Harley D and Rafelski J, Particle Accelerators 37/38, (1991) 409.

[40] Takahashi H, Konts H J C, Grand P, Powell J R and Steinberg M, Atomkernenergie-Kerntechnit 36, (1980) 195.

[41] Menshikor I I and Ponomarev L I: JETP Lett. 46, (1987) 312.

Menshikor L I: "Muon Catalytic Processes in a Dense Low- Temperature Plana" Institute Atomnoj Energii im. I.B. Kurchatova IAE-4589/2 preprint March (1988).

[42] Jändel M, Froelich P, Lasson G and Stodden C D, Phys. Rev. A10, (1989) 2799.

[43] (a) Harley D, Müller B, and Rafelski J, J. Phys. G: Nucl. Part. Phys. 16, (1989) 281.

(b) Harley D, Müller B, and Rafeloki J: "MuCF with Z > 1," in Muon Catalyzed Fusion 1988, AIP Proceedings Seriea No. 181, p 239, New York, 1989. 
[44] Jones S E: "Some surprises in muon catalyzed fusion", in Atomic Physics 9, eds. van Dyck R S and Fortson E N, p 99, Singapore, 1984.

Jones S E: "Survey of Experimental Results in Muon-Catalysed Fusion" in Muon Catalyzed Fusion 1988, AIP Proceedings Series No. 181, p 394, New York, 1989.

[45] Nagamine K, Matsusaki T, Ishida K, Hirata Y, Watanabe Y, Miyake Y, and Kadono R: "Status of Muon-Catalyzed Fusion Experiments at UT-MSL/KEK in Muon Catalyzed Fusion 1988, AIP Proceedings Series No. 181, p 23, New York, 1989.

[46] (a) Jones S E, Palmer E P, Csirr J B, Decker D L, Jensen G L, Thorne J M, Taylor S F, and Rafelski J), Nature 388, (1989) 737.

(b) Jones S E, Palmer E P, Csirr J B, Decker D L, Jensen G L, Thorne J M, Taybor S F, and Rafelski J), Fusion Technology 19, (1990) 199.

[47] Guinan M W, Chapline G F, and Moir R W, "Catalysis of Deuterium Fusion In Metal Hydrides by Coomic Ray Muons" UCRI-100881, preprint (1981).

This suggestion has been criticised by: Cohen J C and Davies J D, Nature 388, (1989) 705.

[48] Zweig G, Science 201, (1978) 973.

[49] Sawicki M, Gajda M, Harley D, and Rafelski J, Fusion Technology 18, (1990) 136.

[50] Mamyrin B A, Khabarin L V and Yudenich V S, Soviet Phys. Dokl. 28, (1978) 581.

[51] DeRujula A, Glashow S L, and Sarid U, Nucl. Phys. 3s3, (1990) 173.

[52] Dimopoulos S, Eichler D, Eomailsadeh R, and Starkman G D, Phys. Rev. D41, (1990) 2388.

[53] Sawicki M, Gajda M, Harley D, and Rafelski J, Phys. Rev. A44, (1991) 4345.

[54] Ioffe B L, Okun L B, Shifman M A, and Voloshin M B, Acta Physica Polonica B12, (1981) 229.

[55] Boyd R N, Takahashi K, Perry R J, and Miller T A, Science 244, (1989) 1450.

[56] Sawicki M and Rafelski J, J. Phys. G16, (1990) L197.

[57] Shin G R and Rafeloki J, Nucl. Instruments and Methods A387, (1990) 565.

[58] Petrov Yu V, Nature 285, (1980) 466.

Petrov Yu V, Muon Catalysed Fusion 2, (1988) 525.

[59] Rafelski J, unpublished.

[60] Van Siclen C D W and Jones S E, J. Phys. G: Nucl. Part. Phys. 12, (1986) 213.

[61] Gajda M, Harley D, and Rafelski J: "Limite on Cold Fusion in Condensed Matter: a Parametric Study", University of Arisona Report AZPH-TH/89-19-2 (broadly circulated, unpublished).

[62] Burrows A, Phys. Rev B40, (1989) 3405.

[63] Żakowics W and Rafeloki J: "Coupled Channel Model for Ultra-Low Energy Deuteron-Deuteron Fusion", in AIP proceedings series, Vol. 228 (1991) p 911, S. E. Jones et al, eds.

[64] (a) Harley D, Gajda M, and Rafelski J: "Review of the Current Status of Cold Fusion" in The Nuclear Equation of State: Discovery of Nuclear Shock Waves and the EOS, W. Greiner and H. Stöcker eds. (Plenum, New York, 1990), p 541.

(b) Harley D, Gajda M, and Rafelski J: "Review of the Current Theoretical Status of Cold Fusion" in Emerging Nuclear Energy Systems 1989, U. von Möllendorf and B. Goel, eds. (World Scientific, Singapore, 1989) p 308.

[65] Horowits C J, Phys. Rev. C10, (1989) R1555. 
[66] (a) Gajda M and Rafelski J, J. Phys. G: Nucl. Part. Phys. 17, (1991) 653.

(b) Gajda M and Rafeloki J: "Nuclear Fusion in Jupiter", in AIP proceedings series, Vol. 228 (1991) P 591, S. E. Jonee et al, eds.

[67] Hubbard W B and Marley M S, Icarus 78, (1989) 102.

[68] Hubbard W B, Ap. J. 152, (1968) 745.

[69] Graboske H G Jr., Pollack J B, Grossman A S, and Olness R J, Ap. J., 199, (1975) 265.

\section{LIST OF FIGURES}

1 Experimental and theoretical final sticking fraction as a function of density. . . . . . 6

2 Representation of $d-t-\mu$ fusion cycle processes . . . . . . . . . . . . . . 8

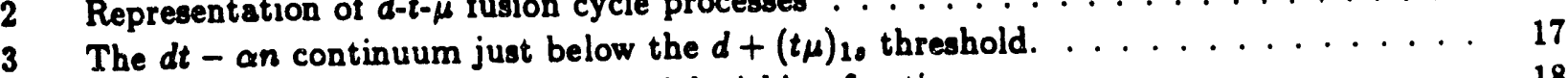

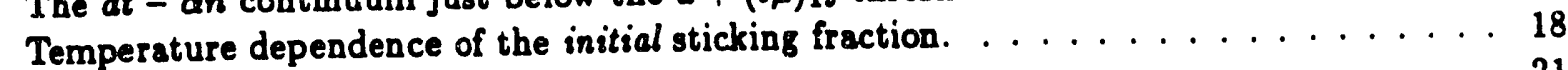

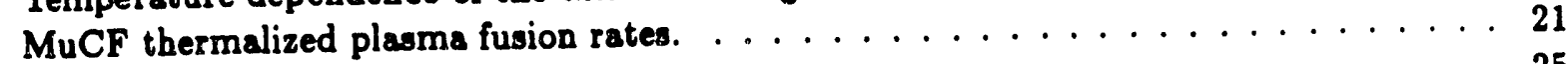

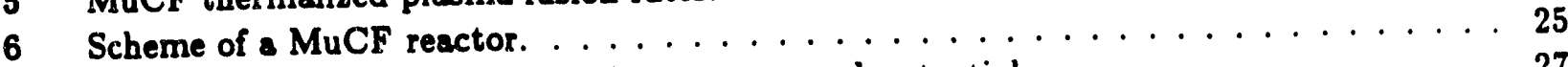

$7 d(d, n){ }^{3} \mathrm{He}$ cross section for an electron screened potential. . . . . . . . . . . 27

\section{LIST OF TABLES}

Hydrogen fusion reactions The $(\alpha \mu)^{+}$sticking fraction for different $d-t$ target densities $\phi=\rho_{0} / \rho_{0} \ldots \ldots \ldots$

Reactivation coefficients of the $(\alpha \mu)^{+}$reaction $R$ as a function of density with quenching cross section and without quenching $(Q=0) \ldots \ldots \ldots \ldots \ldots$ 

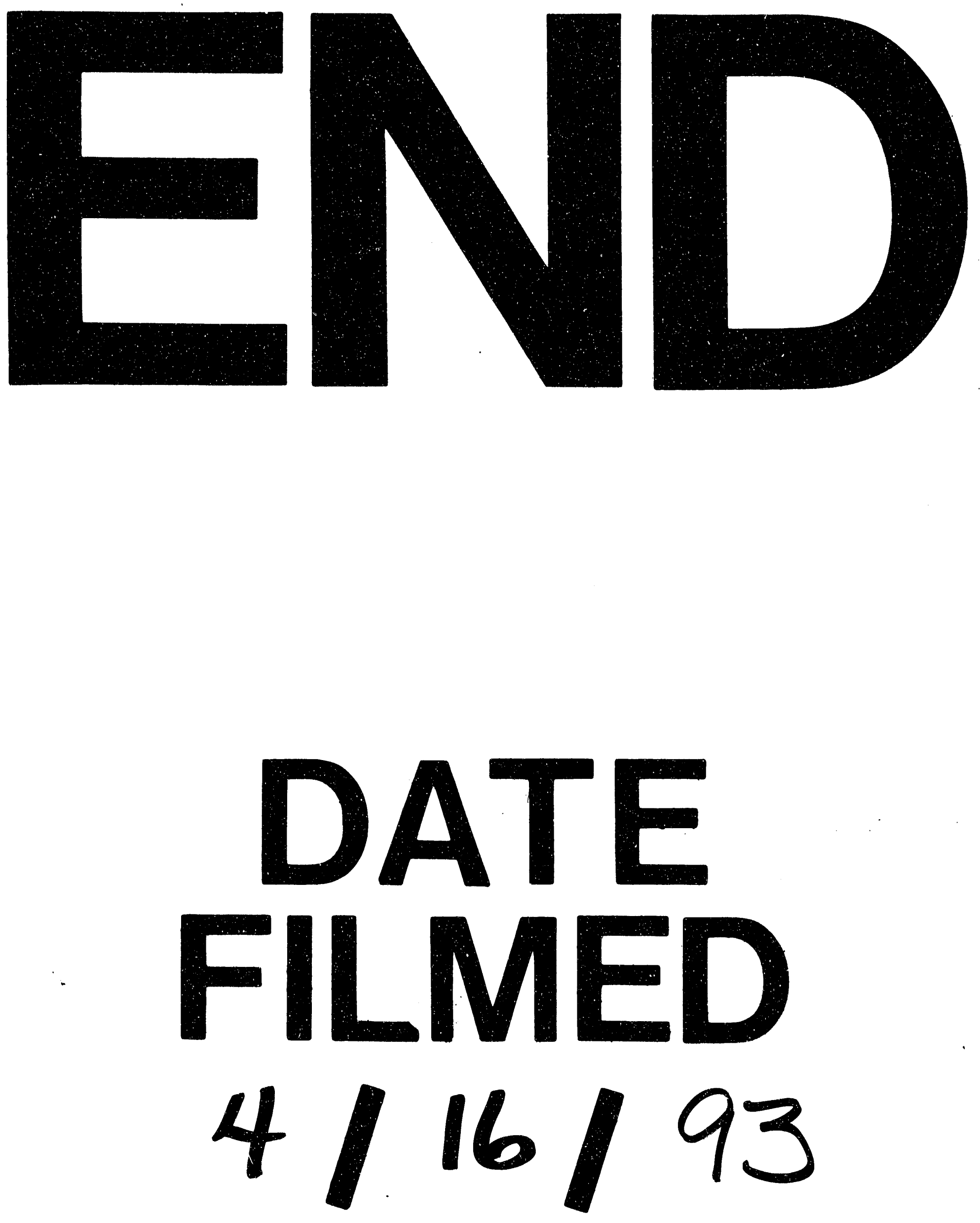
